\title{
HIGHER ORDER OPERATIONS
}

\author{
BY \\ E. SPANIER(1)
}

Introduction. In a recent paper [14] the author gave a general definition of secondary operations $\langle f, g, h\rangle$ which included as special cases various examples of secondary operations which had been considered previously $[1 ; 3 ; 9 ; 11 ; 18]$. This provided a unified treatment of these particular operations and their properties. As pointed out in [14], however, not all examples of secondary operations were included in this treatment. Specifically, cohomology operations involving cup products (such as Steenrod's functional cup product [17] and the Massey triple product [6]) were not obtainable as special cases of the operation $\langle f, g, h\rangle$.

The present paper contains a definition and discussion of operations of all orders. The definition is such that for secondary operations it is more general than the definition in [14] and includes, in addition to the operation $\langle f, g, h\rangle$, examples of secondary operations involving cup products.

The definition of a higher order operation we adopt is that it is a higher order obstruction to the existence of a null-homotopy of a map carried by a carrier on a simplicial complex. Thus a higher order operation will be defined only when certain lower order operations vanish and, when defined, will have a certain indeterminacy. Naturality properties of obstructions imply those of operations, and the fact that obstructions are cocycles gives relations among operations. Various examples of operations are obtained by specializing to particular simplicial complexes, particular carriers on them, and particular maps carried by these carriers.

We present no applications herein of the operations. Many applications of special operations can be found in the literature (for example in $[1 ; 6 ; 10 ; 17$; 18]), and it is our intention to present a unification of the theory of operations rather than new applications of them. However, new secondary operations based on the suspension and loop functors are defined (see (4.4)-(4.7) below) which should have interesting applications. Special cases of the one involving suspension have been considered by Toda in connection with his toral construction (see [19]).

After a first section devoted to notation and some standard facts, the second section deals with coefficient stacks on simplicial complexes and their corre-

Received by the editors November 5, 1962.

(1) Research supported by the Miller Institute for Basic Research and the National Science Foundation. 
sponding cohomology theories. The third section discusses carriers on simplicial complexes and some of their associated stacks. Higher order operations are defined in the fourth section as obstructions to a null-homotopy of a map carried by a carrier. Examples of these operations are given in this section, and in the fifth section basic properties are established. The sixth section is devoted to the operation $\left\langle a_{1}, a_{2}, \cdots, a_{n}\right\rangle$ defined for certain sequences of mappings

$$
A_{0} \stackrel{a_{1}}{\longrightarrow} A_{1} \stackrel{a_{2}}{\longrightarrow} \cdots \stackrel{a_{n}}{\longrightarrow} A_{n}
$$

and which is a straightforward generalization of the operation $\langle f, g, h\rangle$ defined in [14].

1. Preliminaries. The theory of higher order operations we shall develop will be valid for a suitable category. Rather than trying to formulate abstractly the conditions on the category necessary to define the operations we shall work with some specific categories both to develop the general theory and for examples. There are three such specific categories to which we shall refer, namely:

(i) The category of topological spaces with base points (called based spaces) and continuous maps between them.

(ii) The category of quasi-topological spaces with base points and quasicontinuous maps between them [15].

(iii) The category of chain complexes and chain transformations between them [4].

The definitions will usually be given for the first category but hold equally well in each of the other two. The examples will usually involve either the second or third categories.

Let $I$ be the closed unit interval $[0,1]$. If $X$ is a topological space with base point $x_{0}$, we let $T X$ be the cone over $X$ (so $T X$ is the identification space of $X \times I$ in which $X \times 0 \cup x_{0} \times I$ has been collapsed to one point to be used as base point of $T X)$. We regard the points of $T X$ to be parametrized by pairs $(x, t)$ with $x \in X, t \in I$ and identify $X$ with $X \times 1 \subset T X$. Then $S X$ is defined to be the quotient space of $T X$ in which $X$ has been collapsed to a single point which is the base point of $S X$. For $p \geqq 2$ we define $S^{p} X$ inductively by $S^{p} X=S\left(S^{p-1} X\right)$.

Given a map $f: X \rightarrow Y$ between based spaces, the mapping cone $Z_{f}$ is the quotient space of the disjoint union of $T X$ and $Y$ by the identifications $(x, 1) \in T X$ equals $f(x) \in Y$. Then we have an inclusion map $i: Y \subset Z_{f}$ and a quotient map $k: Z_{f} \rightarrow S X$ and a sequence

$$
X \stackrel{f}{\longrightarrow} Y \stackrel{i}{\rightarrow} Z_{f} \stackrel{k}{\longrightarrow} S X \stackrel{S f}{\longrightarrow} S Y \stackrel{S i}{\longrightarrow} S Z_{f} \stackrel{S k}{\longrightarrow} S^{2} X \longrightarrow \cdots .
$$

If $Q$ is any based space we can form the sequence of homotopy classes and induced mappings

$$
[X, Q] \stackrel{f^{\#}}{\longleftarrow}[Y, Q] \stackrel{i^{\#}}{\longleftarrow}\left[Z_{f}, Q\right] \stackrel{k^{\#}}{\longleftarrow}[S X, Q] \stackrel{(S f)^{\#}}{\longleftarrow}[S Y, Q] \stackrel{(S i)^{\#}}{\longleftarrow} \ldots
$$

and this sequence is functorial in $Q$ and exact $[13 ; 14]$. 
All of the above remarks apply equally well to all three of our categories. Our remarks below about loop spaces will only be used in $\$ 6$ for the category of based spaces.

If $Y$ is a topological space with base point $y_{0}$ we let $P Y$ be the space of paths in $Y$ beginning at $y_{0}$ topologized by the compact-open topology, and we let $p: P Y \rightarrow Y$ be the continuous map defined by $p(\omega)=\omega(1)$ for $\omega \in P Y$. Then $P Y$ is a fiber space over $Y$ with fiber $\Omega Y$ the loop space on $Y$. If $Q$ is a topological space, a map $f: Q \rightarrow Y$ is null-homotopic if and only if there is a map $F^{\prime}: Q \rightarrow P Y$ such that $p F^{\prime}=f$. In fact, the set of null-homotopies of $f$ is in canonical 1-1 correspondence with the set of such liftings $F^{\prime}$ as is easily seen.

Let $f: X \rightarrow Y$ be a map between based spaces. The mapping fiber space $E(f)$ is defined by

$$
E(f)=\{(x, \omega) \in X \times P Y \mid f(x)=p(\omega)\} .
$$

$E(f)$ is the induced fiber space of $P Y$ under $f$ so there is a fiber projection $p: E(f) \rightarrow X$ (defined by $p(x, \omega)=x)$ and the fiber is $\Omega Y$. If we have a commutative diagram of continuous maps between based spaces

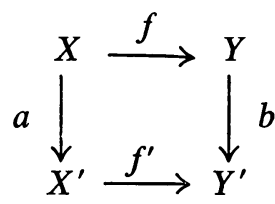

there is a canonical continuous map $c: E(f) \rightarrow E\left(f^{\prime}\right)$ defined by $c(x, \omega)=(a(x),(P b)(\omega))$ for $(x, \omega) \in E(f)$ (where $P b: P Y \rightarrow P Y^{\prime}$ is defined by $((P b)(\omega))(t)=b(\omega(t)))$.

If $Q$ is a topological space, the set of continuous maps $g: Q \rightarrow E(f)$ is clearly in 1-1 correspondence with the set of pairs $\left(g^{\prime}, G^{\prime}\right)$ where $g^{\prime}: Q \rightarrow X, G^{\prime}: Q \rightarrow P Y$ are continuous maps such that $f g^{\prime}=p G^{\prime}$. Hence, the set of continuous maps $g: Q \rightarrow E(f)$ is in 1-1 correspondence with the set of pairs $\left(p g, H^{\prime}\right)$ where $p g: Q \rightarrow X$ and $H^{\prime}$ is a null-homotopy of $f p g$.

Let $K$ be an abstract simplicial complex. Let $\Lambda$ be the set of all finitely nonzero functions $\lambda$ from the vertices of $K$ to the closed unit interval $I$ such that

$$
\sum_{v} \lambda(v)=1 \text {. }
$$

For each simplex $s$ of $K$ let $|s|=\{\lambda \in \Lambda \mid$ if $v$ is not a vertex of $s$ then $\lambda(v)=0\}$. $|s|$ is a metric space with metric

$$
d\left(\lambda, \lambda^{\prime}\right)=\left(\sum_{v \in s}\left(\lambda(v)-\lambda^{\prime}(v)\right)^{2}\right)^{1 / 2} .
$$

The geometric realization $|K|$ is defined to be the subset of $\Lambda$ consisting of the union of $|s|$ for all $s \in K$ and is topologized by the weak topology relative to the subspaces $|s|$. 
Clearly, if $L$ is a subcomplex of $K$, then $|L|$ is a subspace of $|K|$. When we are considering carriers of quasi-topological spaces we shall regard the geometric realization $|K|$ as a quasi-topological space in the quasi-topology associated to the above topology. When dealing with carriers of chain complexes we shall understand by $|K|$ the chain complex generated by the oriented cells of $K$. We use the notation $\left\{v_{0}, v_{1}, \cdots, v_{p}\right\}$ to denote the simplex whose vertices are $v_{0}, v_{1}, \cdots, v_{p}$, and $\left(v_{0}, v_{1}, \cdots, v_{p}\right)$ to denote the oriented cell determined by the ordering $v_{0}, v_{1}, \cdots, v_{p}$ of the vertices $v_{i}$.

Given based topological spaces $X, Y$ we let $C^{\prime}(X, Y)$ denote the quasi-topological space consisting of the continuous maps (preserving base points) $X \rightarrow Y$ with the quasi-topology defined by the condition that if $C$ is a compactHausdorff space, a map $C \rightarrow C^{\prime}(X, Y)$ is quasi-continuous if and only if the corresponding map of $C \times X$ into $Y$ is continuous ( $\$ 4$ of [15]). If $X$ is a locally compact Hausdorff space, this quasi-topology is associated to the compactopen topology on the function space, but for more general spaces it need not be associated to any topology on the set of continuous maps (see (5.5), (5.6) of [15]).

2. Stacks. The theory of obstructions to the extension of mappings and homotopies carried by a carrier leads to cohomology with a general coefficient stack as coefficients $(\$ 13$ of [16]). In this section we define the concept of stack and the corresponding cohomology groups.

Let $K$ be an abstract simplicial complex. $K$ is a partially-ordered set whose elements are the simplexes $s$ of the complex with $s_{1} \leqq s_{2}$ if and only if the simplex $s_{1}$ is a face of the simplex $s_{2}$. A stack $(\Gamma, \gamma)$ of abelian groups on $K$ is a covariant functor from the partially-ordered set $K$ to the category of abelian groups. That is, $\Gamma$ is a function which assigns to each $s \in K$ an abelian group $\Gamma(s)$ and $\gamma$ is a function which assigns to each ordered pair $s_{1} \leqq s_{2}$ in $K$ a homomorphism $\gamma\left(s_{1}, s_{2}\right): \Gamma\left(s_{1}\right) \rightarrow \Gamma\left(s_{2}\right)$ such that:

(2.1) For any $s \in K, \gamma(s, s)$ is the identity map of $\Gamma(s)$.

(2.2) If $s_{1} \leqq s_{2}$ and $s_{2} \leqq s_{3}$, then

$$
\gamma\left(s_{1}, s_{3}\right)=\gamma\left(s_{2}, s_{3}\right) \gamma\left(s_{1}, s_{2}\right) .
$$

Because such a stack involves a covariant functor it, more properly perhaps, ought to be called a covariant stack (as in $\$ 13$ of [16]), but we shall not have occasion in the present paper to refer to the dual concept of a contravariant stack so our terminology should not lead to confusion. We shall also consider stacks of groups (not necessarily abelian) and stacks of sets.

EXAMPLE (2.3). For any abelian group (or non-abelian group or set) $G$ there is a corresponding constant stack on $K$ which assigns to every simplex $s \in K$ the group $G$ and to every ordered pair $s_{1} \leqq s_{2}$ in $K$ the identity map of $G$. 
Example (2.4). Let $K_{1}$ be a subcomplex of a complex $K_{2}$ and let $h$ be a homomorphism of one abelian group $G_{1}$ into another $G_{2}$. Then there is a stack on $K_{2}$ which assigns $G_{1}$ to every simplex in $K_{1}, G_{2}$ to every simplex of $K_{2}-K_{1}$, and to every ordered pair of simplexes $s_{1} \leqq s_{2}$ of $K_{2}$ it assigns either the appropriate identity map or the homomorphism $h$.

Clearly, Example (2.4) can be generalized to larger collections of subcomplexes and groups. Our next example involves the topology of $|K|$.

EXAMPLe (2.5). Let $q$ be a fixed non-negative integer and let $v_{0}$ be a fixed vertex of $K$. To every $p$-simplex $s \in K$ we associate the $q$ th homotopy group $\pi_{q}\left(\left|K^{p}\right|, v_{0}\right)$ of the $p$-dimensional skeleton $K^{p}$ of $K$ with base point $v_{0}$ and to every ordered pair $s_{1} \leqq s_{2}$ of $K$ we associate the corresponding injection of homotopy groups. For $q \geqq 2$ we obtain examples of stacks of abelian groups, while for $q=1$ we have a stack of groups (possibly non-abelian), and for $q=0$ we obtain an example of a stack of sets with base points or zero elements.

The importance of the concept of a stack of abelian groups stems from the fact that such a stack serves as coefficient domain for forming cochain and cohomology groups. Thus, given a stack $(\Gamma, \gamma)$ of abelian groups on $K$ for $p \geqq 0$ a p-cochain $c$ of $K$ with coefficients in $(\Gamma, \gamma)$ is a function which assigns to every oriented $p$-cell $\sigma=\left(v_{0}, v_{1}, \cdots, v_{p}\right)$ of a $p$-simplex $\left\{v_{0}, v_{1}, \cdots, v_{p}\right\}$ of $K$ an element $c(\sigma) \in \Gamma\left(\left\{v_{0}, v_{1}, \cdots, v_{p}\right\}\right)$.If $c_{1}, c_{2}$ are $p$-cochains with coefficients in the same stack, their sum $c_{1}+c_{2}$ is the $p$-cochain defined by

$$
\left(c_{1}+c_{2}\right)(\sigma)=c_{1}(\sigma)+c_{2}(\sigma)
$$

for all oriented $p$-cells $\sigma$ of $K$. In this way the $p$-cochains with coefficients in $(\Gamma, \gamma)$ form an abelian group denoted by $C^{p}(K ;(\Gamma, \gamma))$. For $p<0$ we define $C^{p}(K ;(\Gamma, \gamma))=0$ so that we have cochain groups defined for all integers $p$. Note that the function $\gamma$ plays no role in defining these cochain groups. It will enter now in defining the coboundary homomorphism

$$
\delta: C^{p}(K ;(\Gamma, \gamma)) \rightarrow C^{p+1}(K ;(\Gamma, \gamma)) .
$$

If $\sigma=\left(v_{0}, v_{1}, \cdots, v_{p+1}\right)$ is an oriented $(p+1)$-cell, let $\sigma^{i}$, for $0 \leqq i \leqq p+1$, be the oriented $p$-cell defined by $\sigma^{i}=\left(v_{0}, v_{1}, \cdots, \hat{v}_{i}, \cdots, v_{p+1}\right)$ and let $\gamma_{i}$ be the homomorphism of $\Gamma\left\{v_{0}, v_{1}, \cdots, \hat{v}_{i}, \cdots, v_{p+1}\right\}$ to $\Gamma\left\{v_{0}, v_{1}, \cdots v_{p+1}\right\}$ defined by the functor $\gamma$. For $p \geqq 0$ we define the coboundary homomorphism by the formula

$$
(\delta c)(\sigma)=\sum_{0 \leqq i \leqq p+1}(-1)^{i} \gamma_{i}\left(c\left(\sigma^{i}\right)\right) \text {. }
$$

In the usual way using (2.2) it is easily verified that $\delta \delta=0$. We let $C^{*}(K ;(\Gamma, \gamma))$ denote the cochain complex formed by the cochain groups $C^{p}(K ;(\Gamma, \gamma))$ and the coboundary homomorphism $\delta$, and the cohomology group $H^{p}(K ;(\Gamma, \gamma))$ is defined as usual as the quotient of the kernel of $\left.\delta: C^{p}(K ;(\Gamma, \gamma)) \rightarrow C^{p+1}(K ; \Gamma, \gamma)\right)$ by the image of $\delta: C^{p-1}(K ;(\Gamma, \gamma)) \rightarrow C^{p}(K ;(\Gamma, \gamma))$. Therefore, corresponding to 
a stack $(\Gamma, \gamma)$ of abelian groups we can define cohomology groups $H^{p}(K ;(\Gamma, \gamma))$ for all $p$ and these are abelian groups.

If $(\Gamma, \gamma)$ is the constant stack corresponding to an abelian group $G$ (as in (2.3)), the corresponding cohomology groups are the usual cohomology groups $H^{p}(K ; G)$ of $K$ with coefficients in $G$. In (2.4) if $K_{1} \subset K_{2}$ and we take for $h$ the trivial homomorphism of the zero group into $G$, the corresponding cohomology groups are the usual relative cohomology groups $H^{p}\left(K_{2}, K_{1} ; G\right)$; whereas if we take $h$ to be the trivial homomorphism of $G$ to the zero group, the corresponding cohomology groups are the usual cohomology groups $H^{p}\left(K_{1} ; G\right)$ of $K_{1}$ with coefficients in $G$.

If $(\Gamma, \gamma)$ is a stack of groups (not necessarily abelian), we can define a group $H^{0}(K ;(\Gamma, \gamma))$ and a set with base point $H^{1}(K ;(\Gamma, \gamma))$ but not, in general, $H^{p}(K ;(\Gamma, \gamma))$ for $p>1$ (see also [7]). The $p$-cochains are defined as for the abelian case above and form a group $C^{p}(K ;(\Gamma, \gamma))$ under pointwise multiplication (i.e., if $c_{1}, c_{2}$ are $p$-cochains so is $c_{1} c_{2}$ defined by $\left.\left(c_{1} c_{2}\right)(\sigma)=c_{1}(\sigma) c_{2}(\sigma)\right)$. For $p=0,1$ we define maps

$$
\delta: C^{p}(K ;(\Gamma, \gamma)) \rightarrow C^{p+1}(K ;(\Gamma, \gamma))
$$

as follows: if $\left(v_{0}, v_{1}\right)$ is an orientation of the 1-simplex $s$ and if $c \in C^{0}(K ;(\Gamma, \gamma))$ we set

$$
(\delta c)\left(v_{0}, v_{1}\right)=\left(\gamma\left(v_{0}, s\right) c\left(v_{0}\right)\right)^{-1}\left(\gamma\left(v_{1}, s\right) c\left(v_{1}\right)\right)
$$

while if $\left(v_{0}, v_{1}, v_{2}\right)$ is an orientation of the 2-simplex $s$ and if $s_{i}$ is the face of $s$ opposite $v_{i}$, then for $c \in C^{1}(K ;(\Gamma, \gamma))$ we set

$$
(\delta c)\left(v_{0}, v_{1}, v_{2}\right)=\left(\gamma\left(s_{0}, s\right) c\left(v_{1}, v_{2}\right)\right)\left(\gamma\left(s_{1}, s\right) c\left(v_{0}, v_{2}\right)\right)^{-1}\left(\gamma\left(s_{2}, s\right) c\left(v_{0}, v_{1}\right)\right) .
$$

Then $\delta \delta$ maps $C^{0}(K ;(\Gamma, \gamma))$ into the zero element of $C^{2}(K ;(\Gamma, \gamma))$. Unfortunately, neither map $\delta$ is a homomorphism of the group structures; however, $C^{0}(K ;(\Gamma, \gamma))$ can be made to act on $C^{1}(K ;(\Gamma, \gamma))$ in such a way as to be compatible with $\delta$.

For $d \in C^{1}(K ;(\Gamma, \gamma)), c \in C^{0}(K ;(\Gamma, \gamma))$ we define $d * c \in C^{1}(K ;(\Gamma, \gamma))$ by the condition that if $\left(v_{0}, v_{1}\right)$ is an orientation of the 1-simplex $s$ then

$$
(d * c)\left(v_{0}, v_{1}\right)=\left(\gamma\left(v_{0}, s\right) c\left(v_{0}\right)\right)^{-1} d\left(v_{0}, v_{1}\right)\left(\gamma\left(v_{1}, s\right) c\left(v_{1}\right)\right) .
$$

The following are easily verified:

(2.6) $\left(d * c_{1}\right) * c_{2}=d *\left(c_{1} c_{2}\right)$ for $c_{1}, c_{2} \in C^{0}, d \in C^{1}$.

(2.7) $\delta\left(c_{1} c_{2}\right)=\delta c_{1} * c_{2}$ for $c_{1}, c_{2} \in C^{0}$.

(2.8) $\delta(d * c)=0$ for $c \in C^{0}, d \in C^{1}$ and $\delta d=0$.

(2.9) $0 * c_{1}=0$ for $c_{1} \in C^{0}$ and $\delta c_{1}=0$.

It follows from (2.7) and (2.9) and the fact that $c^{-1}(\sigma)=(c(\sigma))^{-1}$ that those elements of $C^{0}$ which are mapped to 0 by $\delta: C^{0} \rightarrow C^{1}$ form a subgroup of $C^{0}$, and this subgroup is defined to be $H^{0}(K ;(\Gamma, \gamma))$. It follows from $(2.6)$ and 
(2.8) that $C^{0}$ operates on the right on the set of elements of $C^{1}$ sent to 0 by $\delta: C^{1} \rightarrow C^{2}$. The set of orbits of this action of $C^{0}$ on the set mapped to 0 by $\delta: C^{1} \rightarrow C^{2}$ is defined to be $H^{1}(K ;(\Gamma, \gamma))$, which is a set with zero element the orbit of the zero 1-chain.

If $(\Gamma, \gamma)$ is a stack of sets with base points, we can define $H^{0}(K ;(\Gamma, \gamma))$ to be a set with base point but there is, in general, no definition of $H^{p}(K ;(\Gamma, \gamma))$ for $p \geqq 1 . H^{0}(K ;(\Gamma, \gamma))$ is defined to be the set of functions $c$ defined on vertices $v$ of $K$ such that $c(v) \in \Gamma(v)$ and for any 1 -simplex $s \in K$ with vertices $v_{0}, v_{1}$ then

$$
\gamma\left(v_{0}, s\right) c\left(v_{0}\right)=\gamma\left(v_{1}, s\right) c\left(v_{1}\right) .
$$

Stacks of non-abelian groups or stacks of sets with base points are not as interesting as stacks of abelian groups but are discussed here because they occur in the applications of stacks we consider later.

If $(\Gamma, \gamma)$ and $\left(\Gamma^{\prime}, \gamma^{\prime}\right)$ are stacks of abelian groups on the same complex $K$, a homomorphism $h$ from $(\Gamma, \gamma)$ to $\left(\Gamma^{\prime}, \gamma^{\prime}\right)$ is a collection of homomorphisms $h(s): \Gamma(s) \rightarrow \Gamma^{\prime}(s)$ for $s \in K$ such that for every ordered pair $s_{1} \leqq s_{2}$ in $K$ the following diagram is commutative

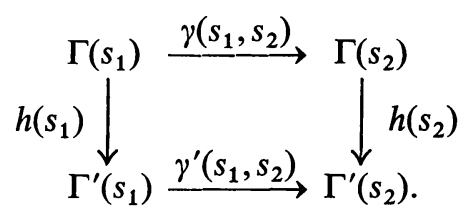

Such a homomorphism $h$ induces homomorphisms

$$
h_{*}: H^{p}(K ;(\Gamma, \gamma)) \rightarrow H^{p}\left(K ;\left(\Gamma^{\prime}, \gamma^{\prime}\right)\right)
$$

so we see that the groups $H^{p}(K ;(\Gamma, \gamma))$ are covariant functors of the stack $(\Gamma, \gamma)$.

If $\mu: K^{\prime} \rightarrow K$ is a simplicial map and if $(\Gamma, \gamma)$ is a stack of abelian groups on $K$, there is an induced stack $(\Gamma \mu, \gamma \mu)$ on $K^{\prime}$ defined by $(\Gamma \mu)\left(s^{\prime}\right)=\Gamma\left(\mu\left(s^{\prime}\right)\right)$ for $s^{\prime} \in K^{\prime}$ and if $s_{1}^{\prime} \leqq s_{2}^{\prime}$ in $K^{\prime}$, then $\mu\left(s_{1}^{\prime}\right) \leqq \mu\left(s_{2}^{\prime}\right)$ in $K$ and we set

$$
\gamma \mu\left(s_{1}^{\prime}, s_{2}^{\prime}\right)=\gamma\left(\mu\left(s_{1}^{\prime}\right), \mu\left(s_{2}^{\prime}\right)\right) .
$$

Such a simplicial map $\mu$ induces homomorphisms

$$
\mu^{*}: H^{p}(K ;(\Gamma, \gamma)) \rightarrow H^{p}\left(K^{\prime} ;(\Gamma \mu, \gamma \mu)\right)
$$

which make the groups $H^{p}(K ;(\Gamma, \gamma))$ contravariant functors of the complex $K$.

If $\left(\Gamma^{\prime}, \gamma^{\prime}\right),(\Gamma, \gamma)$ are stacks of abelian groups on the same complex $K$, we say $\left(\Gamma^{\prime}, \gamma^{\prime}\right)$ is a substack of $(\Gamma, \gamma)$ (denoted by $\left.\left(\Gamma^{\prime}, \gamma^{\prime}\right) \subset(\Gamma, \gamma)\right)$ if for every $s \in K$ the group $\Gamma^{\prime}(s)$ is a subgroup of $\Gamma(s)$ and if this collection of inclusion homomorphisms is a homomorphism from $\left(\Gamma^{\prime}, \gamma^{\prime}\right)$ to $(\Gamma, \gamma)$. In this case we can define the quotient stack $\left(\Gamma^{\prime \prime}, \gamma^{\prime \prime}\right)$ of $(\Gamma, \gamma)$ by $\left(\Gamma^{\prime}, \gamma^{\prime}\right)$ by the condition that $\Gamma^{\prime \prime}(s)=\Gamma(s) / \Gamma^{\prime}(s)$ 
with the maps $\gamma^{\prime \prime}\left(s_{1}, s_{2}\right)$ defined by passing to the quotient with the maps $\gamma\left(s_{1}, s_{2}\right)$. The inclusion map $\left(\Gamma^{\prime}, \gamma^{\prime}\right) \rightarrow(\Gamma, \gamma)$ induces a monomorphism $C^{*}\left(K ;\left(\Gamma^{\prime}, \gamma^{\prime}\right)\right)$ $\rightarrow C^{*}(K ;(\Gamma, \gamma))$, and the quotient map $(\Gamma, \gamma) \rightarrow\left(\Gamma^{\prime \prime}, \gamma^{\prime \prime}\right)$ induces an epimorphism $C^{*}(K ;(\Gamma, \gamma)) \rightarrow C^{*}\left(K ;\left(\Gamma^{\prime \prime}, \gamma^{\prime \prime}\right)\right)$, and we have the following short exact sequence of cochain complexes

$$
0 \rightarrow C^{*}\left(K ;\left(\Gamma^{\prime}, \gamma^{\prime}\right)\right) \rightarrow C^{*}(K ;(\Gamma, \gamma)) \rightarrow C^{*}\left(K ;\left(\Gamma^{\prime \prime}, \gamma^{\prime \prime}\right)\right) \rightarrow 0 .
$$

It follows in the standard way that there is an exact sequence of cohomology groups

$$
\cdots \rightarrow H^{p}\left(K ;\left(\Gamma^{\prime}, \gamma^{\prime}\right)\right) \rightarrow H^{p}(K ;(\Gamma, \gamma)) \rightarrow H^{p}\left(K ;\left(\Gamma^{\prime \prime}, \gamma^{\prime \prime}\right)\right) \rightarrow \cdots .
$$

Let $(\Gamma, \gamma)$ be a stack of abelian groups on $K$ and let $L$ be a subcomplex of $K$. Let $\left(\Gamma^{\prime}, \gamma^{\prime}\right)$ be the stack on $K$ equal to $(\Gamma, \gamma)$ on $K-L$ and to 0 on $L$ and let $\left(\Gamma^{\prime \prime}, \gamma^{\prime \prime}\right)$ be the stack on $K$ equal to $(\Gamma, \gamma)$ on $L$ and to 0 on $K-L$. Then $\left(\Gamma^{\prime}, \gamma^{\prime}\right)$ is a substack of $(\Gamma, \gamma)$ (but $\left(\Gamma^{\prime \prime}, \gamma^{\prime \prime}\right)$ is not a substack even though $\Gamma^{\prime \prime}(s) \subset \Gamma(s)$ for every $s \in K$ because if $s_{1} \leqq s_{2}$ with $s_{1} \in L, s_{2} \in K-L, \gamma\left(s_{1}, s_{2}\right)$ may not be the trivial map of $\Gamma\left(s_{1}\right)$ into $\Gamma\left(s_{2}\right)$ while $\gamma^{\prime \prime}\left(s_{1}, s_{2}\right)$ is necessarily the trivial map of $\Gamma^{\prime \prime}\left(s_{1}\right)=\Gamma\left(s_{1}\right)$ into $\left.\Gamma^{\prime \prime}\left(s_{2}\right)=0\right)$, and $\left(\Gamma^{\prime \prime}, \gamma^{\prime \prime}\right)$ is the quotient stack of $(\Gamma, \gamma)$ by $\left(\Gamma^{\prime}, \gamma^{\prime}\right)$. We define

$$
H^{p}\left(L ;(\Gamma, \gamma)=H^{p}\left(K ;\left(\Gamma^{\prime \prime}, \gamma^{\prime \prime}\right)\right) \text { and } H^{p}(K, L ;(\Gamma, \gamma))=H^{p}\left(K ;\left(\Gamma^{\prime}, \gamma^{\prime}\right)\right) .\right.
$$

It then follows from the exactness of (2.10) that we have the following exact sequence

$$
\cdots \rightarrow H^{p}(K, L ;(\Gamma, \gamma)) \rightarrow H^{p}(K ;(\Gamma, \gamma)) \rightarrow H^{p}(L ;(\Gamma, \gamma)) \rightarrow \cdots
$$

which is a generalization of the exact cohomology sequence of the pair $(K, L)$ to which $(2.11)$ reduces when $(\Gamma, \gamma)$ is a constant stack.

3. Carriers. In this section we define what we shall mean by a carrier and develop some of the theory of maps and homotopies carried by such a carrier. Our definition is somewhat more general than the definition in [16] in that the carriers we consider do not all take values in one topological space.

Let $K$ be an abstract simplicial complex as in $\S 2$. A carrier $(\Phi, \phi)$ of based topological spaces on $K$ is a covariant functor from $K$ to the category of based spaces. That is, $\Phi$ is a function which assigns to each simplex $s \in K$ a based space $\Phi(s)$ and $\phi$ is a function which assigns to each ordered pair $s_{1} \leqq s_{2}$ in $K$ a continuous map (preserving base points) $\phi\left(s_{1}, s_{2}\right): \Phi\left(s_{1}\right) \rightarrow \Phi\left(s_{2}\right)$ such that:

(3.1) For any $s \in K, \phi(s, s)$ is the identity map of $\Phi(s)$.

(3.2) If $s_{1} \leqq s_{2}$, and $s_{2} \leqq s_{3}$, then

$$
\phi\left(s_{1}, s_{3}\right)=\phi\left(s_{2}, s_{3}\right) \phi\left(s_{1}, s_{2}\right) .
$$


Thus, a carrier of based spaces on $K$ is the same as a stack of based spaces on $K$.We prefer to use both terms rather than just one to emphasize a difference in their usage. With a stack on $K$ we shall be mainly interested in the cohomology groups of $K$ with coefficients in the stack as defined in $\$ 2$; whereas with a carrier on $K$ we shall be mainly interested in the mappings and homotopies carried by the carrier, concepts which we define below. We can also consider carriers of quasi-topological spaces with base points and carriers of chain complexes. The only requisite is that continuous map and homotopy be suitably interpreted in each case (for chain complexes they will mean chain transformation and chain homotopy).

EXAMPLE (3.3). For any topological space $X$ with base point there is a constant carrier on $K$ which assigns to each simplex $s \in K$ the space $X$ and to each ordered pair $s_{1} \leqq s_{2}$ of $K$ the identity map of $X$.

EXAmple (3.4). Let $K_{1}$ be a subcomplex of a complex $K_{2}$ and let $\phi$ be a continuous map of one based space $X_{1}$ into another $X_{2}$ which preserves base points. There is then a carrier on $K_{2}$ which assigns to each simplex of $K_{1}$ the space $X_{1}$, to each simplex of $K_{2}-K_{1}$ the space $X_{2}$, and to every ordered pair $s \leqq s_{2}$ of $K_{2}$ either the appropriate identity map or the map $\phi$.

Given a carrier $(\Phi, \phi)$ of based spaces on $K$ a map $f$ carried by $(\Phi, \phi)$ is function which assigns to every simplex $s \in K$ a continuous map

$$
f(s):|s| \rightarrow \Phi(s)
$$

such that for every ordered pair $s_{1} \leqq s_{2}$ in $K$

$$
f\left(s_{2}\right)|| s_{1} \mid=\phi\left(s_{1}, s_{2}\right) f\left(s_{1}\right) .
$$

There is a distinguished map carried by $(\Phi, \phi)$ called the zero map (or trivial map) which assigns to each $s \in K$ the constant map of $|s|$ to the base point of $\Phi(s)$. If $f_{1}, f_{2}$ are two maps carried by $(\Phi, \phi)$ a homotopy $F$ from $f_{1}$ to $f_{2}$ carried by $(\Phi, \phi)$ is a function which assigns to every $s \in K$ a continuous map

$$
F(s):|s| \times I \rightarrow \Phi(s)
$$

which is a homotopy from $f_{1}(s)$ to $f_{2}(s)$ such that for every $s_{1} \leqq s_{2}$ in $K$

$$
F\left(s_{2}\right)|| s_{1} \mid \times I=\phi\left(s_{1}, s_{2}\right) F\left(s_{1}\right) .
$$

If there is a homotopy from $f_{1}$ to $f_{2}$ carried by $(\Phi, \phi)$, we say $f_{1}$ is homotopic to $f_{2}$. This is an equivalence relation in the set of maps carried by $(\Phi, \phi)$ and the corresponding equivalence classes are called homotopy classes. A null-homotopy of $f$ is a homotopy from $f$ to the zero map. If such a homotopy exists, $f$ is said to be null-homotopic.

Given a carrier $(\Phi, \phi)$ on $K$ there are associated stacks $\left(\Gamma_{i}, \gamma_{i}\right)$ on $K$ for $i \geqq 0$ where $\Gamma_{i}(s)=\pi_{i}(\Phi(s))$ for $s \in K$ and for $s_{1} \leqq s_{2}$ in $K$, 


$$
\gamma_{i}\left(s_{1}, s_{2}\right)=\phi\left(s_{1}, s_{2}\right)_{\text {\# }}: \pi_{i}\left(\Phi\left(s_{1}\right)\right) \rightarrow \pi_{i}\left(\Phi\left(s_{2}\right)\right)
$$

is the homomorphism induced by $\phi\left(s_{1}, s_{1}\right)$. Note that the stacks $\left(\Gamma_{i}, \gamma_{i}\right)$ are stacks of abelian groups if $i \geqq 2$, while $\left(\Gamma_{1}, \gamma_{1}\right)$ is a stack of groups and $\left(\Gamma_{0}, \gamma_{0}\right)$ is a stack of sets with base points.

If $X$ and $Y$ are based spaces, then $\pi_{i}\left(C^{\prime}(X, Y)\right) \approx\left[S^{i} X, Y\right][15]$ and if $C$ is a chain complex, then $\pi_{i}(C)$ (which is, by definition, the group of chain homotopy classes of chain transformations of the chain complex of the $i$-dimensional $\mathrm{s}^{\cdot}$ eleton of an $(i+1)$-simplex into $\left.C\right)$ is isomorphic to $H_{i}(C)$ as is easily verified by direct computation.

4. Higher order operations. In this section we define what we shall mean by a higher order operation and present some examples. Our definition is that such an operation is just an obstruction to the construction of a null-homotopy, over the successive skeleta of a complex $K$, of a map $f$ carried by a carrier on $K$.

Let $(\Phi, \phi)$ be a carrier on $K$ and let $f$ be a map carried by it. To each vertex $v \in K$ the map $f$ assigns $f(v) \in \Phi(v)$. Let $\left(\Gamma_{0}, \gamma_{0}\right)$ be the stack of sets with base points with $\Gamma_{0}(s)=\pi_{0}(\Phi(s))$ for $s \in K$, and let $O^{\circ}(f)$ be the zero-dimensional cochain on $K$ with coefficients in $\left(\Gamma_{0}, \gamma_{0}\right)$ defined by the condition that, for a vertex $v \in K,\left(O^{0}(f)\right)(v)$ is the element of $\pi_{0}(\Phi(v))$ determined by $f(v)$. It is a standard verification that $O^{\circ}(f)$ is a cocycle and that it vanishes if and only if $f$ is null-homotopic on $K^{0}$. The cocycle $O^{0}(f)$ is an element of $H^{0}\left(K ;\left(\Gamma_{0}, \gamma_{0}\right)\right)$ and is called the first obstruction to a null-homotopy of $f$. Its vanishing is necessary and sufficient for the existence of a null-homotopy on the zero-dimensional skelton of $K$. This first obstruction corresponds to a general primary operation, but we shall not pursue this viewpoint further now. The higher obstructions are more interesting, and we have discussed the first one only for completeness.

For the second obstruction we assume that $f$ is carried by a carrier $(\Phi, \phi)$ and that $O^{0}(f)$ vanishes so that there is a null-homotopy of $f$ on $K^{0}$. Let $F$ be a nullhomotopy of $f$ on $K^{0}$. Let $\left(v_{0}, v_{1}\right)$ be an orientation of a 1 -simplex $s$ of $K$. We then have a closed path (at the base point)

$$
\left(\phi\left(v_{0}, s\right)\left(F \mid v_{0} \times I\right)^{-1}\right) f\left(v_{0}, v_{1}\right)\left(\phi\left(v_{1}, s\right)\left(F \mid v_{1} \times I\right)\right)
$$

in $\Phi(s)$. Let $\left(\Gamma_{1}, \gamma_{1}\right)$ be the stack of groups where $\Gamma_{1}(s)=\pi_{1}(\Phi(s))$ for $s \in K$. Then there is a 1-dimensional cochain $c^{1}$ of $K$ with coefficients in $\left(\Gamma_{1}, \gamma_{1}\right)$ defined by the condition that $c^{1}\left(v_{0}, v_{1}\right)$ is the homotopy class of the closed path above. It is a standard verification that $\delta c^{1}=0$ and that, though $c^{1}$ depends on the choice of the null-homotopy $F$, the set of cochains $c^{1}$ obtained from all possible choices of null-homotopies $F$ is exactly the same as the set of cocycles in a cohomology class of $C^{1}\left(K ;\left(\Gamma_{1}, \gamma_{1}\right)\right)$ (i.e., it is an orbit of $C^{1}\left(K ;\left(\Gamma_{1}, \gamma_{1}\right)\right)$ under the action of $\left.C^{0}\left(K ;\left(\Gamma_{1}, \gamma_{1}\right)\right)\right)$. Therefore, $f$ determines a well-defined element $O^{1}(f)$ $\in H^{1}\left(K ;\left(\Gamma_{1}, \gamma_{1}\right)\right)$ which is the set of all the cocycles $c^{1}$ above, and it is easy to verify 
as usual that $f$ is null-homotopic on $K^{1}$ if and only if $O^{1}(f)$ is the zero element of $H^{1}\left(K ;\left(\Gamma_{1}, \gamma_{1}\right)\right)$. Note that $O^{1}(f)$ is defined if and only if $O^{0}(f)$ vanishes. It is called the second obstruction to a null-homotopy of $f$, and from our point of view will be a secondary operation.

Before considering higher obstructions we discuss some examples of second obstructions whose corresponding secondary operations are familiar. This will provide some justification for our definition of operations as obstructions. It is worth noting that by considering the simplest possible complex on which second obstructions can occur, namely, the complex consisting of a single 1-simplex with its two vertices, one obtains interesting nontrivial examples of secondary operations. In the examples (4.1)-(4.7) below we shall let $K$ denote this complex consisting of a single 1-simplex $s$ with its two vertices $v, v^{\prime}$.

EXAMPLE (4.1). In [14] the author studied secondary operations arising from a sequence $A \stackrel{a}{\rightarrow} B \stackrel{b}{\rightarrow} C \stackrel{c}{\rightarrow} D$ in which $A, B, C, D$ are based spaces and $b a, c b$ are both assumed to be null-homotopic. The corresponding secondary operation $\langle a, b, c\rangle$ was a double coset of $[S A, D]$ under left multiplication by an element of $c_{\#}[S A, C]$ and right multiplication by an element of $(S a) \#[S B, D]$. We shall show how $\langle a, b, c\rangle$ can be identified with a second obstruction to a null-homotopy of a suitable map on $K$.

Let $(\Phi, \phi)$ be the carrier on $K$ defined by:

$$
\Phi(s)=C^{\prime}(A, D), \Phi(v)=C^{\prime}(A, C), \Phi\left(v^{\prime}\right)=C^{\prime}(B, D)
$$

and the maps

$$
\begin{array}{ll}
\phi(v, s)(\alpha)=c \alpha & \text { for } \alpha \in C^{\prime}(A, C), \\
\phi\left(v^{\prime}, s\right)(\beta)=\beta a & \text { for } \beta \in C^{\prime}(B, D) .
\end{array}
$$

We define a map $f$ on $K$ carried by $(\Phi, \phi)$ by:

$$
f(v)=b a \in \Phi(v), \quad f\left(v^{\prime}\right)=c b \in \Phi\left(v^{\prime}\right)
$$

$f(s)$ is the constant map of $|s|$ to $c b a \in \Phi(s)$.

Then $f$ is null-homotopic on $K^{0}$ if and only if $b a$ and $c b$ are null-homotopic. Hence, the condition that the first obstruction to a null-homotopy of $f$ vanish is exactly the same as the condition that $\langle a, b, c\rangle$ be defined. If this first obstruction vanishes, there is defined a second obstruction $O^{1}(f) \in H^{1}\left(K ;\left(\Gamma_{1}, \gamma_{1}\right)\right)$. The definition of $O^{1}(f)$ shows that any representative cocycle $c^{1}$ of it takes values on the two orientations of $s$ which are inverse to each other so such a cocycle is determined by its value on the orientation $\left(v, v^{\prime}\right)$ of $s$. Now $c^{1}\left(v, v^{\prime}\right)$ is an element of $\pi_{1}\left(C^{\prime}(A, D)\right)$, which is isomorphic to [SA,D], and given a cochain $c^{0} \in C^{0}\left(K ;\left(\Gamma_{1}, \gamma_{1}\right)\right)$ (so $c^{0}(v) \in \pi_{1}\left(C^{\prime}(A, C)\right) \approx[S A, C]$ and $c^{0}\left(v^{\prime}\right) \in \pi_{1}\left(C^{\prime}(B, D)\right)$ $\approx[S B, D])$, then 


$$
\left(c^{1} * c_{0}\right)\left(v, v^{\prime}\right)=\left(c\left(c^{0}(v)\right)^{-1}\right) c^{1}\left(v, v^{\prime}\right)\left(c^{0}\left(v^{\prime}\right)(S a)\right) .
$$

Since $c^{0}(v), c^{0}\left(v^{\prime}\right)$ are arbitrary, the values of the cochains $c^{1}$ representing $O^{1}(f)$ on $\left(v, v^{\prime}\right)$ form a double coset of $[S A, D]$ determined up to left multiplication by an element of $c_{\#}[S A, C]$ and right multiplication by an element of $(S a)^{\#}[S B, D]$. It is easy to verify directly from the definitions that the second obstruction $O^{1}(f)$ corresponds in this way to the secondary operation $\langle a, b, c\rangle$.

From example (4.1) it follows [14] that the toral construction, functional cohomology operations, and secondary cohomology operations are all obtained as second obstructions to a null-homotopy of a suitable map on $K$. Our next two examples show how secondary operations involving the cup product can also be obtained as second obstructions.

EXAMPle (4.2) (The FUnCTIONAL CUP PRODUCT). We start with a continuous map $g: X \rightarrow Y$ and two singular cohomology classes $u_{1} \in H^{p}\left(Y ; G_{1}\right), u_{2} \in H^{q}\left(Y ; G_{2}\right)$. The functional cup product [17] denoted by $u_{1} \cup_{g} u_{2}$ is defined if $g^{*} u_{1}=0$ and $u_{1} \cup u_{2}=0$ (as an element of $H^{p+q}\left(Y ; G_{3}\right)$ where $G_{1}, G_{2}$ are paired to $\left.G_{3}\right)$ and is an element of $H^{p+q-1}\left(X ; G_{3}\right)$ modulo the subgroup generated by $H^{p-1}\left(X ; G_{1}\right)$ $\cup g^{*} u_{2}$ and $g^{*}\left(H^{p+q-1}\left(Y ; G_{3}\right)\right)$.

We shall construct a carrier $(\Phi, \phi)$ of chain complexes on $K$ and a map $f$ carried by $(\Phi, \phi)$ such that the first obstruction to a null-homotopy of $f$ vanishes if and only if the functional cup product above is defined and, in this case, the second obstruction to a null-homotopy of $f$ will correspond to this functional cup product.

If $C^{*}$ is a cochain complex with a homogeneous coboundary operator $\delta$ of degree +1 , we let ${ }_{n} C^{*}$ denote the chain complex defined by $\left({ }_{n} C^{*}\right)_{m}=\left(C^{*}\right)^{n-m}$ with boundary operator $\partial$ of degree -1 equal to $\delta$. Let $C^{*}(X ; G)$ be the singular cochain complex of $X$ with coefficients in $G$. The continuous map $g: X \rightarrow Y$ induces a cochain transformation $g^{*}: C^{*}(Y ; G) \rightarrow C^{*}(X ; G)$ for any $G$. Let $u_{1}^{\prime} \in C^{p}\left(Y ; G_{1}\right), u_{2}^{\prime} \in C^{q}\left(Y ; G_{2}\right)$ be representative cocycles of $u_{1}, u_{2}$, respectively. We define a carrier $(\Phi, \phi)$ of chain complexes on $K$ by:

$$
\Phi(v)={ }_{p+q} C^{*}\left(Y ; G_{3}\right), \quad \Phi\left(v^{\prime}\right)={ }_{p} C^{*}\left(X ; G_{1}\right), \quad \Phi(s)={ }_{p+q} C^{*}\left(X ; G_{3}\right)
$$

and $\phi(v, s)=g^{*}, \phi\left(v^{\prime}, s\right)=$ cup product on the right by $g^{*} u_{2}^{\prime}$. Note that $\phi(v, s)$ and $\phi\left(v^{\prime}, s\right)$ are then homogeneous chain transformations of degree 0 . Let $f$ be the map carried by $(\Phi, \phi)$ defined by:

$$
f(v)=u_{1}^{\prime} \cup u_{2}^{\prime}, \quad f\left(v^{\prime}\right)=g^{*} u_{1}^{\prime}
$$

$f(s)$ sends $\left(v, v^{\prime}\right)$ to 0 and both $v$ and $v^{\prime}$ to $g^{*} u_{1}^{\prime} \cup g^{*} u_{2}^{\prime}$.

The condition that the first obstruction to a null-homotopy of $f$ vanish is that $u_{1}^{\prime} \cup u_{2}^{\prime}$ and $g^{*} u_{1}^{\prime}$ both be cohomologous to zero which, in turn, is the condition that the functional cup product be defined. If this is so, the second obstruction 
to a null-homotopy of $f$ is completely determined by its value on the orientation $\left(v, v^{\prime}\right)$ of $s$, and this value is an element of $\pi_{1}\left({ }_{p+q} C^{*}\left(X ; G_{3}\right)\right) \approx H^{p+q-1}\left(X ; G_{3}\right)$ determined modulo $\phi(v, s) \pi_{1}\left({ }_{p+4} C^{*}\left(Y ; G_{3}\right)\right) \approx g^{*} H^{p+q-1}\left(Y ; G_{3}\right)$ and

$$
\phi\left(v^{\prime}, s\right) \pi_{1}\left({ }_{p} C^{*}\left(X ; G_{1}\right)\right) \approx H^{p-1}\left(X ; G_{1}\right) \cup g^{*} u_{2} .
$$

Therefore, the second obstruction to a null-homotopy of $f$ has the same indeterminacy as $u_{1} \cup_{g} u_{2}$, and it is easy to verify that they are, in fact, equal.

EXAmple (4.3). (The Massey TRIPLE PRODUCT). In this case we are given $u_{1} \in H^{p}\left(X ; G_{1}\right), u_{2} \in H^{q}\left(X ; G_{2}\right), u_{3} \in H^{r}\left(X ; G_{3}\right)$ with $G_{1}, G_{2}$ paired to $G_{12}$ and $G_{12}, G_{3}$ paired to $G_{123}$ and also $G_{2}, G_{3}$ paired to $G_{23}$ and $G_{1}, G_{23}$ paired to $G_{123}$ so that the following diagram of these pairing maps is commutative

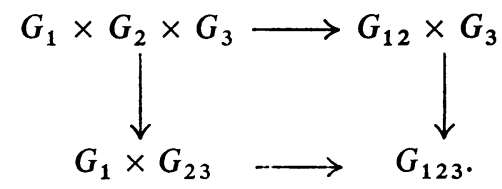

The Massey triple product is defined if $u_{1} \cup u_{2}=0$ and $u_{2} \cup u_{3}=0$ and is then an element of $H^{p+q+r-1}\left(X ; G_{123}\right)$ modulo the subgroup generated by $u_{1} \cup H^{q+r-1}\left(X ; G_{23}\right)$ and $H^{p+q-1}\left(X ; G_{12}\right) \cup u_{3}$.

To obtain the Massey triple product as a second obstruction to a null-homotopy of a map $f$ carried by a carrier $(\Phi, \phi)$ let $u_{i}^{\prime}$ be a representative cocycle of $u_{i}$ for $i=1,2,3$. Let $(\Phi, \phi)$ be the carrier of chain complexes on $K$ defined by:

$$
\begin{aligned}
\Phi(v) & ={ }_{p+q} C^{*}\left(X ; G_{12}\right), \quad \Phi\left(v^{\prime}\right)={ }_{q+r} C^{*}\left(X ; G_{23}\right), \\
\Phi(s) & ={ }_{p+q+r} C^{*}\left(X ; G_{123}\right), \\
\phi(v, s) & =\text { cup product on the right by } u_{3}^{\prime}, \\
\phi\left(v^{\prime}, s\right) & =\text { cup product on the left by } u_{1}^{\prime} .
\end{aligned}
$$

Let $f$ be the map carried by $(\Phi, \phi)$ defined by:

$$
f(v)=u_{1}^{\prime} \cup u_{2}^{\prime}, \quad f\left(v^{\prime}\right)=u_{2}^{\prime} \cup u_{3}^{\prime},
$$

$f(s)$ maps $\left(v, v^{\prime}\right)$ to 0 and both $v$ and $v^{\prime}$ to $u_{1}^{\prime} \cup u_{2}^{\prime} \cup u_{3}^{\prime}$.

The first obstruction to a null-homotopy vanishes if and only if $u_{1}^{\prime} \cup u_{2}^{\prime}$ and $u_{2}^{\prime} \cup u_{3}^{\prime}$ are both cohomologous to 0 , and this is the condition that the Massey triple product be defined. If this is so, the second obstruction $O^{1}(f)$ is defined and is easily identified with the Massey triple product.

The next four examples of secondary operations seem to be new.

EXAmple (4.4). Suppose we are given continuous maps $A \stackrel{a}{\rightarrow} B \stackrel{b}{\rightarrow} C$ between based spaces such that $b a$ is null-homotopic and the suspension $S b: S B \rightarrow S C$ is also null-homotopic. There is then a secondary operation which 
associates to the data an element of $\left[S^{2} A, S C\right]$ defined modulo the subgroup generated by $\left(S^{2} a\right)^{\#}\left[S^{2} B, S C\right]$ and $S([S A, C])$. To define this secondary operation we define a carrier $(\Phi, \phi)$ on $K$ by:

$$
\Phi(s)=C^{\prime}(S A, S C), \Phi(v)=C^{\prime}(A, C), \Phi\left(v^{\prime}\right)=C^{\prime}(S B, S C)
$$

and the maps

$$
\begin{aligned}
\phi(v, s)(\alpha)=S(\alpha) & \text { for } \alpha \in C^{\prime}(A, C) \\
\phi\left(v^{\prime}, s\right)(\beta)=\beta S a & \text { for } \beta \in C^{\prime}(S B, S C) .
\end{aligned}
$$

We define a map $f$ carried by $(\Phi, \phi)$ by the conditions:

$$
f(v)=b a, \quad f\left(v^{\prime}\right)=S b,
$$

$f(s)$ is the constant map of $|s|$ to $(S b)(S a)=S(b a)$.

Then $f$ is null-homotopic on $K^{0}$ if and only if $b a$ and $S b$ are both null-homotopic. In this case the second obstruction to a null-homotopy of $f$ is determined by its value on the oriented 1 -simplex $\left(v, v^{\prime}\right)$. This value is an element of $\pi_{1}\left(C^{\prime}(S A, S C)\right) \approx\left[S^{2} A, S C\right]$ defined modulo $\phi(v, s)_{\#} \pi_{1}\left(C^{\prime}(A, C)\right) \approx S[S A, C]$ and $\phi\left(v^{\prime}, s\right)_{\#} \pi_{1}\left(C^{\prime}(S B, S C)\right) \approx\left(S^{2} a\right)^{\#}\left[S^{2} B, S C\right]$, and this is the desired secondary operation. We denote by $\langle a, b, S\rangle$ the element of $\left[S^{2} A, S C\right] /\left(\left(S^{2} a\right)^{\#}\left[S^{2} B, S C\right]+S[S A, C]\right)$ determined by this secondary operation.

EXAMPLE (4.5). Given continuous maps $A \stackrel{a}{\rightarrow} B \stackrel{b}{\rightarrow} C$ between based spaces such that $b a$ is null-homotopic and $S a: S A \rightarrow S B$ is null-homotopic there is defined a secondary operation

$$
\langle S, a, b\rangle \in\left[S^{2} A, S C\right] /\left(S[S A, C]+(S b)_{\text {\# }}\left[S^{2} A, S B\right]\right) .
$$

This is defined similarly to $\langle a, b, S\rangle$ of Example (4.4) so details are omitted.

Similarly, instead of using the suspension functor $S$ we could use the loop functor $\Omega$ to obtain the next two examples. We omit the details.

EXAMPLE (4.6). Given continuous maps $A \stackrel{a}{\rightarrow} B \stackrel{b}{\rightarrow} C$ between based spaces such that $b a$ is null-homotopic and $\Omega b: \Omega B \rightarrow \Omega C$ is null-homotopic there is defined a secondary operation

$$
\left.\langle a, b, \Omega\rangle \in\left[\Omega A, \Omega^{2} C\right] /(\Omega a)^{\#}\left[\Omega B, \Omega^{2} C\right]+\Omega[A, \Omega C]\right) .
$$

EXAMPLE (4.7). Given continuous maps $A \stackrel{a}{\rightarrow} B \stackrel{b}{\rightarrow} C$ between based spaces such that $b a$ is null-homotopic and $\Omega a: \Omega A \rightarrow \Omega B$ is null-homotopic there is defined a secondary operation

$$
\langle\Omega, a, b\rangle \in\left[\Omega A, \Omega^{2} C\right] /\left(\Omega[A, \Omega C]+\left(\Omega^{2} b\right)_{\#}\left[\Omega A, \Omega^{2} B\right]\right) .
$$


Our next example involves a more complicated complex consisting of a 2-simplex and all its faces.

EXAmple (4.8). Consider a sequence

$$
A \stackrel{a}{\longrightarrow} B \stackrel{b}{\longrightarrow} C \stackrel{c}{\longrightarrow} D \stackrel{d}{\longrightarrow} E
$$

of continuous maps between based spaces. Let $\bar{K}$ be the complex consisting of a 2-simplex $t$ with vertices $v, v^{\prime}, v^{\prime \prime}$ and 1-simplexes $s=\left(v^{\prime}, v^{\prime \prime}\right), s^{\prime}=\left(v, v^{\prime \prime}\right)$, $s^{\prime \prime}=\left(v, v^{\prime}\right)$. We define a carrier $(\Phi, \phi)$ of quasi-topological spaces on $\bar{K}$ by:

$$
\begin{aligned}
& \Phi(v)=C^{\prime}(A, C), \quad \Phi\left(v^{\prime}\right)=C^{\prime}(B, D), \quad \Phi\left(v^{\prime \prime}\right)=C^{\prime}(C, E), \\
& \Phi(s)=C^{\prime}(B, E), \quad \Phi\left(s^{\prime}\right)=C^{\prime}(A, E), \quad \Phi\left(s^{\prime \prime}\right)=C^{\prime}(A, D), \\
& \Phi(t)=C^{\prime}(A, E)
\end{aligned}
$$

and the maps $\phi$ all defined by composition on the left or right or both by the appropriate maps $a, b, c, d$; so, for example,

$$
\begin{aligned}
& \phi\left(v, s^{\prime}\right)(\alpha)=d c \alpha \quad \text { for } \alpha \in C^{\prime}(A, C), \\
& \phi\left(v^{\prime}, s^{\prime \prime}\right)(\beta)=\beta a, \phi\left(v^{\prime}, t\right)(\beta)=d \beta a \text { for } \beta \in C^{\prime}(B, D), \\
& \phi\left(v^{\prime \prime}, s^{\prime}\right)(\gamma)=\gamma b a \text { for } \gamma \in C^{\prime}(C, E), \\
& \phi(s, t)(\delta)=\delta a \text { for } \delta \in C^{\prime}(B, E) .
\end{aligned}
$$

We define a map $f$ carried by $(\Phi, \phi)$ by:

$$
f(v)=b a, \quad f\left(v^{\prime}\right)=c b, \quad f\left(v^{\prime \prime}\right)=d c,
$$

$$
f(s), f\left(s^{\prime}\right), f\left(s^{\prime \prime}\right), f(t) \text { are all appropriate constant maps. }
$$

Then $f$ is null-homotopic on $\bar{K}^{0}$ if and only if $b a, c b, d c$ are all null-homotopic. If this is so, let $F$ be a null-homotopy of $f$ on $R^{0}, F$ canj always be extended to a null-homotopy of $f$ on $s^{\prime}$ (because both maps $\phi\left(v, s^{\prime}\right)$ and $\phi\left(v^{\prime \prime}, s^{\prime}\right)$ being compositions with $d c$ and $b a$, respectively, are themselves null-homotopic) so the obstruction to extending $F$ over $R^{1}$ is completely determined by its value on the oriented 1-simplex $\left(v^{\prime}, v^{\prime \prime}\right)$ and its value on the oriented 1-simplex $\left(v, v^{\prime}\right)$. Let $u \in \pi_{1}(\Phi(s)) \approx[S B, E]$ be the obstruction to extending $F$ evaluated on the orientation $\left(v^{\prime}, v^{\prime \prime}\right)$ of $s$ and let $u^{\prime \prime} \in \pi_{1}\left(\Phi\left(s^{\prime \prime}\right)\right) \approx[S A, D]$ be the obstruction to exten$\operatorname{ding} F$ evaluated on the orientation $\left(v, v^{\prime}\right)$ of $s^{\prime \prime}$. Because the obstruction to extending $F$ is a cocycle we find by evaluating its coboundary on the oriented 2-simplex $\left(v, v^{\prime}, v^{\prime \prime}\right)$ that

$$
\left((S a)^{\#} u\right)\left(d_{\#} u^{\prime \prime}\right)=1 \text {. }
$$

Therefore, from a particular $F$ we obtain a pair $u \in[S B, E], u^{\prime \prime} \in[S A, D]$ satisfying (4.9). A different choice of $F$ will give a pair $u_{1}, u_{1}^{\prime \prime}$ related to the above pair $u, u^{\prime \prime}$ by equations 


$$
u_{1}=\left(d_{\#} \beta\right) u\left((S b)^{\#} \gamma\right), \quad u_{1}^{\prime \prime}=\left(c_{\#} \alpha\right) u^{\prime \prime}\left((S a)^{\#} \beta\right)^{-1},
$$

where $\alpha \in[S A, C], \beta \in[S B, D], \gamma \in[S C, E]$. Furthermore, given $\alpha, \beta, \gamma$ as above, there is a choice of the null-homotopy of $f$ on $R^{0}$ such that the corresponding pair $u_{1}, u^{\prime \prime}$ will be related to $u, u^{\prime \prime}$ by (4.10), where $\alpha, \beta, \gamma$ are the specified elements.

Therefore, the secondary operation in this case defines a pair $u, u^{\prime \prime}$ satisfying the single relation (4.9) and with the indeterminacy (4.10). The first element $u$ with its indeterminacy as determined from (4.10) is exactly $\langle b, c, d\rangle$ while the second element $u^{\prime \prime}$, with its indeterminacy is exactly the element $\langle a, b, c\rangle$. However, the pair $u, u^{\prime \prime}$ is determined up to smaller indeterminacy than the two indeterminacies of $\langle b, c, d\rangle$ and $\langle a, b, c\rangle$ taken together (because the same element $\beta \in[S B, D]$ must be used in both $u$ and $u^{\prime \prime}$ in (4.10)). Hence, the vanishing of the pair $u, u^{\prime \prime}$ is a stronger condition than merely the vanishing of $\langle b, c, d\rangle$ and the vanishing of $\langle a, b, c\rangle$. This is to be expected because the vanishing of $u, u^{\prime \prime}$ is equivalent to the existence of a null-homotopy of $f$ on $K^{1}$, and this is stronger than the existence of a null-homotopy of $f$ on each 1-simplex of $\vec{K}$.

Note that (4.9), when $u, u^{\prime \prime}$ are replaced by $\langle b, c, d\rangle,\langle a, b, c\rangle$, respectively, is Theorem (4.5) of [14]. Thus, consideration of the complex $R$ provides a method of obtaining relations among secondary operations which can be defined by means of the complex $K$. For example, Theorems 7.1, 7.2, 8.1, 8.2 of [11] follow (up to sign) by considering suitable carriers on $K$. Similar considerations can be used to prove the following relations.

(4.11) Given a sequence of continuous maps

$$
A \stackrel{a}{\longrightarrow} B \stackrel{b}{\longrightarrow} C \stackrel{c}{\longrightarrow} D
$$

between based spaces such that $S a, b a, c b$ are all null-homotopic, then,

$$
S\langle a, b, c\rangle+(S c)_{\#}\langle S, a, b\rangle=0
$$

in $\left[S^{2} A, S D\right] / S\left(c_{\#}[S A, C]\right)$.

(4.12) Given a sequence of continuous maps

$$
A \stackrel{a}{\longrightarrow} B \stackrel{b}{\longrightarrow} C \stackrel{c}{\longrightarrow} D
$$

between based spaces such that $b a, c b, S c$ are all null-homotopic, then

$$
\left(S^{2} a\right)^{*}\langle b, c, S\rangle+S\langle a, b, c\rangle=0
$$

in $\left[S A^{2}, S D\right] /\left(S^{2} a\right)^{\#}(S[S B, D])$.

(4.13) Given a sequence of continuous maps

$$
A \stackrel{a}{\longrightarrow} B \stackrel{b}{\longrightarrow} C \stackrel{c}{\longrightarrow} D
$$

between based spaces such that $b a, S b, c b$ are all null-homotopic, then 


$$
\left(S^{2} a\right)^{\#}\langle S, b, c\rangle+(S c)_{\#}\langle a, b, S\rangle=0
$$

in $\left[S^{2} A, S D\right] /\left(\left(S^{2} a\right)^{\#}(S c)_{\#}\left[S^{2} B, S C\right]+\left(S^{2} a\right)^{\#} S[S B, D]+(S c)_{\#} S[S A, C]\right)$.

(4.14) Given a sequence of continuous maps

$$
A \stackrel{a}{\longrightarrow} B \stackrel{b}{\longrightarrow} C \stackrel{c}{\longrightarrow} D
$$

between based spaces such that $S(b a), c b a, S(c b)$ are all null-homotopic, then

$$
\langle S a, S b, S c\rangle=\langle S, b a, c\rangle+\langle a, c b, S\rangle
$$

in $\left[S^{2} A, S D\right] /\left(\left(S^{2} a\right)^{\#}\left[S^{2} B, S D\right]+S[S A, D]+(S c)_{\#}\left[S^{2} A, S C\right]\right)$.

There are, of course, relations analogous to those of (4.11)-(4.14) when the suspension functors are replaced by the loop functor $\Omega$.

To define higher order operations we assume that $(\Phi, \phi)$ is a carrier on $K$ and that $f$ is a map carried by it such that for some $p \geqq 1, f \mid K^{p}$ is nullhomotopic. If $F$ is a null-homotopy of $f \mid K^{p}$, there is an obstruction $c^{p+1}(F)$ to extending $F$ to a null-homotopy over $K^{p+1} \cdot c^{p+1}(F)$ is a $(p+1)$-cocycle of $K$ with coefficients in the stack $\left(\Gamma_{p+1}, \gamma_{p+1}\right)$, where $\Gamma_{p+1}(s)=\pi_{p+1}(\Phi(s))$ for $s \in K$. The cocycle $c^{p+1}(F)$ depends on the choice of the null-homotopy $F$, however, if $\left\{F_{j}\right\}$ is the collection of all null-homotopies of $f$ which agree on $K^{p-1}$ with $F$, then it is standard [8] that the collection $\left\{c^{p+1}\left(F_{j}\right)\right\}$ of obstruction cocycles is a cohomology class of $C^{p+1}\left(K ;\left(\Gamma_{p+1}, \gamma_{p+1}\right)\right)$ so corresponds to a unique element of $H^{p+1}\left(K ;\left(\Gamma_{p+1}, \gamma_{p+1}\right)\right)$. Therefore, the collection of obstruction cocycles for all null-homotopies $F$ of $f$ on $K^{p}$ corresponds to some subset $O^{p+1}(f)$ of $H^{p+1}\left(K ;\left(\Gamma_{p+1}, \gamma_{p+1}\right)\right)$. This subset is the $(p+2)$ nd-obstruction to a nullhomotopy of $f$ and $f \mid K^{p+1}$ is null-homotopic if and only if $O^{p+1}(f)$ contains the zero element of $H^{p+1}\left(K ;\left(\Gamma_{p+1}, \gamma_{p+1}\right)\right)$. We shall regard $O^{p+1}(f)$ as an operation of order $p+2$.

Note that $O^{p+1}(f)$ is only defined if $f \mid K^{p}$ is null-homotopic. Thus, $O^{p+1}(f)$ is only defined if $O^{p}(f)$ is defined and contains the zero element of $H^{p}\left(K ;\left(\Gamma_{p}, \gamma_{p}\right)\right)$. In this way we are led to consider the sequence of obstructions $O^{0}(f), O^{1}(f), \cdots$. The first one $O^{0}(f)$ is always defined and is a 0 -dimensional cocycle. If this cocycle is zero, there is a cohomology class $O^{1}(f)$ defined. If this cohomology class is zero, there is defined $O^{2}(f)$ which is a subset of a cohomology group. If this subset contains zero, $O^{3}(f)$ is defined, etc. From the viewpoint of higher order operations the $(p+2)$ nd order operation $O^{p+1}(f)$ is defined if and only if the sequence of lower order operations $O^{0}(f), O^{1}(f), \cdots, O^{p}(f)$ are all defined and each contains the corresponding zero element. We say that an operation $O^{p+1}(f)$ vanishes if it is defined and contains the zero element.

It is also of interest to consider operations which correspond to a map $f$ carried by a carrier $(\Phi, \phi)$ on $K$ and to a fixed null-homotopy $F$ on a subcomplex $L \subset K$. In this case when $O^{p+1}(f, F)$ is defined it is a coset of a subgroup 
$H^{p+1}\left(K, L ;\left(\Gamma_{p+1}, \gamma_{p+1}\right)\right)$ and under the injection $H^{p+1}\left(K, L ;\left(\Gamma_{p+1}, \gamma_{p+1}\right)\right)$ $\rightarrow H^{p+1}\left(K ;\left(\Gamma_{p+1}, \gamma_{p+1}\right)\right)$ the coset $O^{p+1}(f, F)$ maps into a subset of $O^{p+1}(f)$. We do not go into details of this case, but it can be developed without any essential changes.

5. Properties of the operations. Naturality properties of the higher order operations introduced in the last section will be established in this section as well as some additivity properties of these operations.

Let $(\Phi, \phi)$ be a carrier on $K$ and let $\mu$ be a simplicial map of a complex $K^{\prime}$ into $K$. There is an induced carrier $\left(\Phi^{\prime}, \phi^{\prime}\right)$ on $K^{\prime}$ defined by $\Phi^{\prime}\left(s^{\prime}\right)=\Phi\left(\mu\left(s^{\prime}\right)\right)$ for $s^{\prime} \in K^{\prime}$ and if $s_{1}^{\prime} \leqq s_{2}^{\prime}$ in $K^{\prime}$ then $\mu\left(s_{1}^{\prime}\right) \leqq \mu\left(s_{2}^{\prime}\right)$ and

$$
\phi^{\prime}\left(s_{1}^{\prime}, s_{2}^{\prime}\right)=\phi\left(\mu\left(s_{1}^{\prime}\right), \mu\left(s_{2}^{\prime}\right)\right) .
$$

If $\left(\Gamma_{p+1}^{\prime}, \gamma_{p+1}^{\prime}\right)$ is the stack on $K^{\prime}$ associated to $\left(\Phi^{\prime}, \phi^{\prime}\right)$ in the same way that $\left(\Gamma_{p+1}, \gamma_{p+1}\right)$ is associated to $(\Phi, \phi)$, then $\left(\Gamma_{p+1}^{\prime}, \gamma_{p+1}^{\prime}\right)$ equals the induced stack $\left(\Gamma_{p+1} \mu, \gamma_{p+1} \mu\right)$ (defined in $\left.\S 2\right)$ so there is an induced homomorphism

$$
\mu^{*}: H^{p+1}\left(K ;\left(\Gamma_{p+1}, \gamma_{p+1}\right)\right) \rightarrow H^{p+1}\left(K^{\prime} ;\left(\Gamma_{p+1}^{\prime}, \gamma_{p+1}^{\prime}\right)\right) .
$$

If $f$ is a map carried by $(\Phi, \phi)$, then $f \mu$ is a map carried by $\left(\Phi^{\prime}, \phi^{\prime}\right)$, and we would expect some relation such as the following between the obstructions to a nullhomotopy of $f$ and of $f \mu$.

THEOREM (5.1). If $O^{p+1}(f)$ is defined, then $O^{p+1}(f \mu)$ is also defined and

$$
O^{p+1}(f \mu) \supset \mu^{*}\left(O^{p+1}(f)\right) \text {. }
$$

Proof. Since $\mu\left(K^{\prime}\right)^{p} \subset K^{p}$, if $F$ is a null-homotopy of $f$ on $K^{p}$ there is a null-homotopy $F^{\prime}$ of $f \mu$ on $\left(K^{\prime}\right)^{p}$ defined by $F^{\prime}\left(s^{\prime}\right)=F\left(\mu\left(s^{\prime}\right)\right)$ for $s^{\prime} \in\left(K^{\prime}\right)^{p}$. Hence, the first part of the theorem is proved, while the second part follows from the equality

$$
c^{p+1}\left(F^{\prime}\right)=\mu^{*} c^{p+1}(F)
$$

which is a direct consequence of the definition of $c^{p+1}$.

Let $(\Phi, \phi)$ and $\left(\Phi^{\prime}, \phi^{\prime}\right)$ be carriers on the same complex $K$ and let $P$ be a natural transformation from $(\Phi, \phi)$ to $\left(\Phi^{\prime}, \phi^{\prime}\right)$; that is, $P$ assigns to each $s \in K$ a map

$$
P(s): \Phi(s) \rightarrow \Phi^{\prime}(s)
$$

such that if $s_{1} \leqq s_{2}$ in $K$ then

$$
\phi^{\prime}\left(s_{1}, s_{2}\right) P\left(s_{1}\right)=P\left(s_{2}\right) \phi\left(s_{1}, s_{2}\right) .
$$

Let $\left(\Gamma_{p+1}, \gamma_{p+1}\right)$ and $\left(\Gamma_{p+1}^{\prime}, \gamma_{p+1}^{\prime}\right)$ be the associated stacks (where $\Gamma_{p+1}(s)$ $=\pi_{p+1}(\Phi(s))$ and $\Gamma_{p+1}^{\prime}(s)=\pi_{p+1}\left(\Phi^{\prime}(s)\right)$ for $\left.s \in K\right)$. Then $P$ induces a homomorphism $\boldsymbol{P}_{*}$ from $\left(\Gamma_{p+1}, \gamma_{p+1}\right)$ to $\left(\Gamma_{p+1}^{\prime}, \gamma_{p+1}^{\prime}\right)$ and hence, as in $\S 2$, a homomorphism 


$$
P_{*}: H^{p+1}\left(K ;\left(\Gamma_{p+1}, \gamma_{p+1}\right)\right) \rightarrow H^{p+1}\left(K ;\left(\Gamma_{p+1}^{\prime}, \gamma_{p+1}^{\prime}\right)\right) .
$$

If $f$ is a map carried by $(\Phi, \phi)$, then $P f$ is a map carried by $\left(\Phi^{\prime}, \phi^{\prime}\right)$, and the following result relates the obstructions to a null-homotopy of $\boldsymbol{f}$ and $\boldsymbol{P f}$.

THEOREM (5.2). If $O^{p+1}(f)$ is defined, then $O^{p+1}(P f)$ is defined and

$$
O^{p+1}(P f) \supset P_{*} O^{p+1}(f) \text {. }
$$

Proof. If $F$ is a null-homotopy of $f$ on $K^{p}$, then $P F$ is a null-homotopy of $P f$ on $K^{p}$, which proves the first part of the theorem. The second part follows from the equality

which is easily verified.

$$
c^{p+1}(P F)=P_{*} c^{p+1}(F)
$$

The naturality properties of higher order operations expressed by (5.1) and (5.2) apply to the secondary operation $\langle a, b, c\rangle$ of Example (4.1) and [14] and in this case specialize to yield (4.3) of [14] (when the diagram of (4.3) is commutative rather than merely homotopy commutative) and (4.4) of [14]. The other properties of $\langle a, b, c\rangle$ in $\$ 4$ of [14] also have analogues for higher order operations, and we next consider the extent to which a higher order operation is independent of the maps used to define it as long as each is allowed to vary in its homotopy class. First we prove the following extension of (5.2).

Lemma (5.3). Let $P$ a natural transformation from $(\Phi, \phi)$ to $\left(\Phi^{\prime}, \phi^{\prime}\right)$ such that for each $s \in K$ the map $P(s)$ induces isomorphisms

$$
P(s)_{\#}: \pi_{p}(\Phi(s)) \approx \pi_{p}\left(\Phi^{\prime}(s)\right) \text { for all } p .
$$

Then $P_{*}$ is an isomorphism of $H^{p+1}\left(K ;\left(\Gamma_{p+1}, \gamma_{p+1}\right)\right)$ onto $H^{p+1}\left(K ;\left(\Gamma_{p+1}^{\prime}, \gamma_{p+1}^{\prime}\right)\right)$ for all $p$, and if $f$ is a map carried by $(\Phi, \phi)$ such that $O^{p+1}(f)$ is defined, then

$$
O^{p+1}(P f)=P_{*} O^{p+1}(f) .
$$

Proof. If $F^{\prime}$ is a null-homotopy of $P f$ on $K^{p}$, then by step-by-step extension over the successive skeleta of $K$ (using the property that $P(s)$ induces isomorphisms of $\pi_{p}(\Phi(s))$ with $\left.\pi_{p}\left(\Phi^{\prime}(s)\right)\right)$ we can construct a null-homotopy $F$ of $f$ such that $P F$ and $F^{\prime}$ are homotopic. Then

$$
c^{p+1}\left(F^{\prime}\right)=c^{p+1}(P F)=P_{*} c^{p+1}(F)
$$

which shows that $O^{p+1}(P f) \subset P_{*} O^{p+1}(f)$. This with (5.2) gives the desired result.

Lemma (5.4). Let $f_{1}, f_{2}$ be two maps carried by the same carrier $(\Phi, \phi)$ and assume they are homotopic by a homotopy carried by $(\Phi, \phi)$. Then $O^{p+1}\left(f_{1}\right)$ is defined if and only if $O^{p+1}\left(f_{2}\right)$ is defined and, in this case 


$$
O^{p+1}\left(f_{1}\right)=O^{p+1}\left(f_{2}\right) \text {. }
$$

Proof. Let $H$ be a homotopy from $f_{1}$ to $f_{2}$ carried by $(\Phi, \phi)$. If $F_{2}$ is a nullhomotopy of $f_{2}$ on $K^{p}$, then $H \mid K^{p}$ can be combined with $F_{2}$ to give a nullhomotopy $F_{1}$ of $f_{1}$ on $K^{p}$ which is homotopic to $F_{2}$. Then $c^{p+1}\left(F_{1}\right)=c^{p+1}\left(F_{2}\right)$ showing that $O^{p+1}\left(f_{2}\right) \subset O^{p+1}\left(f_{1}\right)$. The opposite inclusion follows by interchanging the roles of $f_{1}$ and $f_{2}$.

Let $(\Phi, \phi)$ and $\left(\Phi, \phi^{\prime}\right)$ be two carriers on $K$ with the same object function $\Phi$. We say $(\Phi, \phi)$ is homotopic to $\left(\Phi, \phi^{\prime}\right)$ if there is a carrier $(\bar{\Phi}, \bar{\phi})$ on $K$ such that for $s \in K$

$$
\bar{\Phi}(s)=\Phi(s) \times I
$$

and for $s_{1} \leqq s_{2}$ in $K$ and $x \in \Phi\left(s_{1}\right)$

$$
\begin{aligned}
& \bar{\phi}\left(s_{1}, s_{2}\right)(x, 0)=\left(\phi\left(s_{1}, s_{2}\right) x, 0\right), \\
& \phi\left(s_{1}, s_{2}\right)(x, 1)=\left(\phi^{\prime}\left(s_{1}, s_{2}\right) x, 1\right) .
\end{aligned}
$$

The carrier $(\bar{\Phi}, \bar{\phi})$ is called a homotopy from $(\Phi, \phi)$ to $\left(\Phi, \phi^{\prime}\right)$. For each $s_{1} \leqq s_{2}$ if $\pi: \Phi\left(s_{2}\right) \times I \rightarrow \Phi\left(s_{2}\right)$ is the projection to the first factor, then the composite

$$
\Phi\left(s_{1}\right) \times I \stackrel{\phi\left(s_{1}, s_{2}\right)}{\longrightarrow} \Phi\left(s_{2}\right) \times I \stackrel{\pi}{\longrightarrow} \Phi\left(s_{2}\right)
$$

is a homotopy from $\phi\left(s_{1}, s_{2}\right)$ to $\phi^{\prime}\left(s_{1}, s_{2}\right)$ so the associated stacks $\left(\Gamma_{p+1}, \gamma_{p+1}\right)$ and $\left(\Gamma_{p+1}, \gamma_{p+1}^{\prime}\right)$ are equal.

If $(\bar{\Phi}, \phi)$ is a homotopy from $(\Phi, \phi)$ to $\left(\Phi, \phi^{\prime}\right)$, the inclusion maps $P, P^{\prime}$ defined by

$$
P(s)(x)=(x, 0), P^{\prime}(s)(x)=(x, 1) \text { for } s \in K, x \in \Phi(s)
$$

are natural transformations $P$ of $(\Phi, \phi)$ to $(\bar{\Phi}, \bar{\phi})$ and $P^{\prime}$ of $\left(\Phi, \phi^{\prime}\right)$ to $(\bar{\Phi}, \bar{\phi})$ such that for each $s \in K$ the maps $P(s)$ and $P^{\prime}(s)$ induce isomorphisms of all homotopy groups. We obtain the following result which is a generalization of (4.1) of [14] and yields (4.3) of [14] with a homotopy commutative diagram.

THEOREM (5.5). Let $f$ be carried by a carrier $(\Phi, \phi)$ and $f^{\prime}$ be carried by a carrier $\left(\Phi, \phi^{\prime}\right)$. Let $(\bar{\Phi}, \bar{\phi})$ be a homotopy from $(\Phi, \phi)$ to $\left(\Phi, \phi^{\prime}\right)$ such that Pf and $P^{\prime} f^{\prime}$ are homotopic in $(\bar{\Phi}, \bar{\phi})$. Then $O^{p+1}(f)$ is defined if and only if $O^{p+1}\left(f^{\prime}\right)$ is defined and, in this case,

$$
O^{p+1}(f)=O^{p+1}\left(f^{\prime}\right)
$$

Proof. As pointed out above, the stacks $\left(\Gamma_{p+1}, \gamma_{p+1}\right)$ associated to $(\Phi, \phi)$ and $\left(\Gamma_{p+1}, \gamma_{p+1}^{\prime}\right)$ associated to $\left(\Phi, \phi^{\prime}\right)$ are equal so $O^{p+1}(f)$ and $O^{p+1}\left(f^{\prime}\right)$ are both subsets of the same cohomology group. Using (5.3), (5.4) we have (if $O^{p+1}(f)$ and $O^{p+1}\left(f^{\prime}\right)$ are both defined) 


$$
P_{*} O^{p+1}(f)=O^{p+1}(P f)=O^{p+1}\left(P^{\prime} f^{\prime}\right)=P_{*}^{\prime} O^{p+1}\left(f^{\prime}\right) \text {. }
$$

If $\left(\bar{\Gamma}_{p+1}, \bar{\gamma}_{p+1}\right)$ is the stack associated to $(\bar{\Phi}, \bar{\phi})$, then $P_{\#}$ is an isomorphism of $\left(\Gamma_{p+1}, \gamma_{p+1}\right)$ with $\left(\bar{\Gamma}_{p+1}, \bar{\gamma}_{p+1}\right)$, and $P_{\#}^{\prime}$ is the same isomorphism of $\left(\Gamma_{p+1}, \gamma_{p+1}^{\prime}\right)$ $=\left(\Gamma_{p+1}, \gamma_{p+1}\right)$ with $\left(\bar{\Gamma}_{p+1}, \bar{\gamma}_{p+1}\right)$. It then follows from the above equation and (5.3) that $O^{p+1}(f)=O^{p+1}\left(f^{\prime}\right)$.

It follows from this equality by an inductive argument that if $O^{p+1}(f)$ is defined, so $O^{0}(f), O^{1}(f), \cdots, O^{p}(f)$ all vanish, then $O^{0}\left(f^{\prime}\right), O^{1}\left(f^{\prime}\right), \cdots, O^{p}\left(f^{\prime}\right)$ all vanish, so $O^{p+1}\left(f^{\prime}\right)$ is defined, and this completes the proof.

We have already pointed out that (4.5) of [14] follows from our equation (4.9). We next investigate additivity properties of the higher order operations as these should correspond to additivity properties of $\langle a, b, c\rangle$ established in $\S 4$ of [14].

If $(\Phi, \phi)$ and $\left(\Phi^{\prime}, \phi^{\prime}\right)$ are carriers on the same complex $K$, we define their pro$\operatorname{duct}\left(\Phi \times \Phi^{\prime}, \phi \times \phi^{\prime}\right)$ to be the carrier on $K$ defined by $\left(\Phi \times \Phi^{\prime}\right)(s)=\Phi(s) \times \Phi^{\prime}(s)$ for $s \in K$ and for $s_{1} \leqq s_{2}$ in $K$

$$
\left(\phi \times \phi^{\prime}\right)\left(s_{1}, s_{2}\right)=\phi\left(s_{1}, s_{2}\right) \times \phi^{\prime}\left(s_{1}, s_{2}\right)
$$

Then we have natural transformations

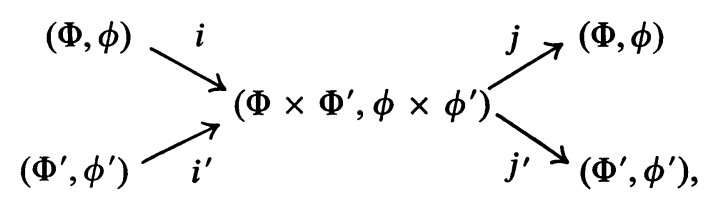

where $i(s)(x)=(x, *)$ for $s \in K, x \in \Phi(s)$ (where * is the base point of $\Phi^{\prime}(s)$ ) $i^{\prime}(s)\left(x^{\prime}\right)=\left(*, x^{\prime}\right)$ for $s \in K, x^{\prime} \in \Phi^{\prime}(s)$ (where * is the basepoint of $\Phi(s)$ ), and $j(s)\left(x, x^{\prime}\right)=x, j^{\prime}(s)\left(x, x^{\prime}\right)=x^{\prime}$ for $s \in K,\left(x, x^{\prime}\right) \in \Phi(s) \times \Phi^{\prime}(s)$. In the corresponding diagram of homotopy groups $\pi_{p+1}\left(\Phi(s) \times \Phi^{\prime}(s)\right)$ is exhibited as the direct sum of $i_{\#} \pi_{p+1}(\Phi(s))$ and $i_{\#}^{\prime} \pi_{p+1}\left(\Phi^{\prime}(s)\right)$. Therefore, the stack of homotopy groups associated to $\left(\Phi \times \Phi^{\prime}, \phi \times \phi^{\prime}\right)$, denoted by $\left(\Gamma_{p+1}^{\prime \prime}, \gamma_{p+1}^{\prime \prime}\right)$, is isomorphic to the direct sum of the stacks $\left(\Gamma_{p+1}, \gamma_{p+1}\right)$ and $\left(\Gamma_{p+1}^{\prime}, \gamma_{p+1}^{\prime}\right)$ so

$$
H^{p+1}\left(K ;\left(\Gamma_{p+1}^{\prime \prime}, \gamma_{p+1}^{\prime \prime}\right)\right) \approx H^{p+1}\left(K ;\left(\Gamma_{p+1}, \gamma_{p+1}\right)\right) \oplus H^{p+1}\left(K ;\left(\Gamma_{p+1}^{\prime}, \gamma_{p+1}^{\prime}\right)\right) \text {. }
$$

Lemma (5.6). Let $f$ be carried by $(\Phi, \phi)$ and $f^{\prime}$ be carried by $\left(\Phi^{\prime}, \phi^{\prime}\right)$. Then $f \times f^{\prime}$ is carried by $\left(\Phi \times \Phi^{\prime}, \phi \times \phi^{\prime}\right)$, and if $O^{p+1}(f), O^{p+1}\left(f^{\prime}\right)$ are both defined, then $O^{p+1}\left(f \times f^{\prime}\right)$ is also defined and

$$
O^{p+1}\left(f \times f^{\prime}\right)=i_{*} O^{p+1}(f)+i_{*}^{\prime} O^{p+1}\left(f^{\prime}\right) .
$$

Proof. If $F$ is a null-homotopy of $f$ on $K^{p}$ and $F^{\prime}$ is a null-homotopy of $f^{\prime}$ on $K^{p}$, then $F \times F^{\prime}$ is a null-homotopy of $f \times f^{\prime}$ on $K^{p}$ proving the first part. Since $c^{p+1}\left(F \times F^{\prime}\right)=i_{*} c^{p+1}(F)+i_{*}^{\prime} c^{p+1}\left(F^{\prime}\right)$ and any null-homotopy of $f \times f^{\prime}$ on $K^{p}$ is of the form $F \times F^{\prime}$, the second part also follows. 
We now assume $L$ is a subcomplex of $K$ and that $\Phi$ is a function on $K$ such that for every $s \in K-L, \Phi(s)$ is an $H$-space. Let $(\Phi, \phi)$ and $\left(\Phi, \phi^{\prime}\right)$ be two carriers with the object function $\Phi$ which agree on $L$ and such that for $s_{1} \leqq s_{2}$ in $K-L$ then $\phi\left(s_{1}, s_{2}\right)=\phi^{\prime}\left(s_{1}, s_{2}\right)$ is a homomorphusm of $\Phi\left(s_{1}\right)$ into $\Phi\left(s_{2}\right)$. We define another carrier $\left(\Phi, \phi^{\prime \prime}\right)$ on $K$ to have the same object function and such that if $s_{1} \leqq s_{2}$ with $s_{1}, s_{2}$ both in $L$ or both not in $L$ then $\phi^{\prime \prime}\left(s_{1}, s_{2}\right)=\phi\left(s_{1}, s_{2}\right)$ $\left(=\phi^{\prime}\left(s_{1}, s_{2}\right)\right)$ while if $s_{1} \leqq s_{2}$ with $s_{1} \in L, s_{2} \in K-L$ then

$$
\phi^{\prime \prime}\left(s_{1}, s_{2}\right)=\phi\left(s_{1}, s_{2}\right) \cdot \phi^{\prime}\left(s_{1}, s_{2}\right) \text {, }
$$

where the right-hand side is the product in the $H$-space structure of $\Phi\left(s_{2}\right)$. Let $f$ be carried by $(\Phi, \phi), f^{\prime}$ be carried by $\left(\Phi, \phi^{\prime}\right)$ and assume $f\left|L=f^{\prime}\right| L$. Define $f^{\prime \prime}$ carried by $\left(\Phi, \phi^{\prime \prime}\right)$ by the condition

$$
\begin{aligned}
& f^{\prime \prime}(s)=f(s)\left(=f^{\prime}(s)\right) \quad \text { for } s \in L, \\
& f^{\prime \prime}(s)=f(s) \circ f^{\prime}(s) \text { for } s \in K-L .
\end{aligned}
$$

It is easy to verify that $f^{\prime \prime}$ is, in fact, a map carried by $\left(\Phi, \phi^{\prime \prime}\right)$. We want to study what relation, if any, there is among $O^{p+1}(f), O^{p+1}\left(f^{\prime}\right)$ and $O^{p+1}\left(f^{\prime \prime}\right)$.

Let $(\Psi, \psi)$ be the carrier on $K$ defined by

$$
\begin{aligned}
& \Psi(s)=\Phi(s) \times \Phi(s) \text { for } s \in L, \\
& \Psi(s)=\Phi(s) \text { for } s \in K-L,
\end{aligned}
$$

and if $s_{1} \leqq s_{2}$ we define

$$
\psi\left(s_{1}, s_{2}\right)=\left\{\begin{array}{l}
\phi\left(s_{1}, s_{2}\right) \times \phi^{\prime}\left(s_{1}, s_{2}\right) \text { if } s_{1}, s_{2} \in L, \\
\phi\left(s_{1}, s_{2}\right)\left(=\phi^{\prime}\left(s_{1}, s_{2}\right)\right) \text { if } s_{1}, s_{2} \in K-L, \\
\phi\left(s_{1}, s_{2}\right) p_{1} \cdot \phi\left(s_{1}, s_{2}\right) p_{2} \text { if } s_{1} \in L, s_{2} \in K-L,
\end{array}\right.
$$

where, for $j=1,2, p_{j}: \Phi(s) \times \Phi(s) \rightarrow \Phi(s)$ is the projection to the $j$ th coordinate.

There are natural transformations $P, P^{\prime}, P^{\prime \prime}$ from $(\Phi, \phi),\left(\Phi, \phi^{\prime}\right),\left(\Phi, \phi^{\prime \prime}\right)$, respectively, to $(\Psi, \psi)$ defined by

$$
\begin{aligned}
& P(s)=P^{\prime}(s)=P^{\prime \prime}(s)=\text { identity map of } \Phi(s) \text { for } s \in K-L, \\
& \left.\begin{array}{l}
P(s)(x)=(x, *) \\
P^{\prime}(s)(x)=(*, x)
\end{array}\right\} \text { for } s \in L, x \in \Phi(s) \text {, where } * \text { is the basepoint of } \Phi(s), \\
& P^{\prime \prime}(s)(x)=(x, x) \text { for } s \in L, x \in \Phi(s) \text {. }
\end{aligned}
$$

THEOREM (5.7). With the notation above if $O^{p+1}(f)$ and $O^{p+1}\left(f^{\prime}\right)$ are both defined, then $O^{p+1}\left(P^{\prime \prime} f^{\prime \prime}\right)$ is defined and

$$
O^{p+1}\left(P^{\prime \prime} f^{\prime \prime}\right) \supset P_{*} O^{p+1}(f)+P_{*}^{\prime} O^{p+1}\left(f^{\prime}\right) .
$$


Proof. Clearly if $F$ is a null-homotopy of $f$ on $K^{p}$ and $F^{\prime}$ is a null-homotopy of $f^{\prime}$ on $K$ we can define a null-homotopy of $F^{\prime \prime}$ of $P^{\prime \prime} f^{\prime \prime}$ on $K^{p}$ by

$$
\begin{aligned}
& F^{\prime \prime}(s)=F(s) \times F^{\prime}(s) \text { for } s \in L, \\
& F^{\prime \prime}(s)=F(s) \cdot F^{\prime}(s) \text { for } s \in K-L .
\end{aligned}
$$

This shows that $O^{p+1}\left(P^{\prime \prime} f^{\prime \prime}\right)$ is defined if both $O^{p+1}(f)$ and $O^{p+1}\left(f^{\prime}\right)$ are defined.

We define a natural transformation $Q$ from $\left(\Phi \times \Phi, \phi \times \phi^{\prime}\right)$ to $(\Psi, \psi)$ by $Q(s)=$ identity map of $\Phi(s) \times \Phi(s)$ for $s \in L$ and $Q(s)=$ multiplication map $\Phi(s) \times \Phi(s) \rightarrow \Phi(s)$ for $s \in K-L$. Then $Q\left(f \times f^{\prime}\right)=P^{\prime \prime} f^{\prime \prime}$. Therefore, by (5.2),

$$
O^{p+1}\left(P^{\prime \prime} f^{\prime \prime}\right)=O^{p+1}\left(Q\left(f \times f^{\prime}\right)\right) \supset Q_{*} O^{p+1}\left(f \times f^{\prime}\right) .
$$

By (5.6), $O^{p+1}\left(f \times f^{\prime}\right)=i_{*} O^{p+1}(f)+i_{*}^{\prime} O^{p+1}\left(f^{\prime}\right)$, and the result follows on observing that $Q i=P, Q i^{\prime}=P^{\prime}$.

COROLlARY (5.8). Let $(\Phi, \phi)$ be a carrier of $H$-spaces and homomorphisms on $K$ and let $f, f^{\prime}$ be maps carried by $(\Phi, \phi)$. Let $f^{\prime \prime}$ be the map $f \cdot f^{\prime}$. Then $f^{\prime \prime}$ is also carried by $(\Phi, \phi)$, and if $O^{p+1}(f), O^{p+1}\left(f^{\prime}\right)$ are both defined, so is $\mathrm{O}^{p+1}\left(f^{\prime \prime}\right)$, and

$$
O^{p+1}\left(f^{\prime \prime}\right) \supset O^{p+1}(f)+O^{p+1}\left(f^{\prime}\right) .
$$

Proof. We apply (5.7) with $L$ the empty subcomplex of $K$. Then $(\Phi, \phi)$, $\left(\Phi, \phi^{\prime}\right),\left(\Phi, \phi^{\prime \prime}\right)$ and $(\Psi, \psi)$ of $(5.7)$ are all equal to $(\Phi, \phi)$, and $P, P^{\prime}, P^{\prime \prime}$ are all the identity transformation of $(\Phi, \phi)$ to itself. Therefore, we have, by (5.7),

$$
O^{p+1}\left(f^{\prime \prime}\right) \supset O^{p+1}(f)+O^{p+1}\left(f^{\prime}\right) \text {. }
$$

(5.7) is a generalization of (4.6), (4.6) of [14] and (5.8) is a generalization of (4.7), $(4.7)^{\prime}$ of [14].

6. The operation $\left\langle a_{1}, a_{2}, \cdots, a_{n}\right\rangle$. In this section we consider particular higher order operations which are straightforward generalizations of the secondary operation $\langle a, b, c\rangle$ of Example (4.1). We begin with the tertiary case.

Let

$$
A_{0} \stackrel{a_{1}}{\longrightarrow} A_{1} \stackrel{a_{2}}{\longrightarrow} A_{2} \stackrel{a_{3}}{\longrightarrow} A_{3} \stackrel{a_{4}}{\longrightarrow} A_{4}
$$

be a sequence of continuous maps between based spaces. Let $K$ be the complex composed of a 2-simplex with vertices $v_{1}, v_{2}, v_{3}$ and all of its faces. We define a carrier $(\Phi, \phi)$ on $K$ by:

$$
\begin{aligned}
\Phi\left(v_{1}\right)=C^{\prime}\left(A_{0}, A_{2}\right), & \Phi\left(v_{2}\right)=C^{\prime}\left(A_{1}, A_{3}\right), \Phi\left(v_{3}\right)=C^{\prime}\left(A_{2}, A_{4}\right), \\
\Phi\left(\left\{v_{1}, v_{2}\right\}\right)=C^{\prime}\left(A_{0}, A_{3}\right), & \Phi\left(\left\{v_{1}, v_{3}\right\}\right)=C^{\prime}\left(A_{0}, A_{2}\right) \times C^{\prime}\left(A_{2}, A_{4}\right), \\
\Phi\left(\left\{v_{2}, v_{3}\right\}\right)=C^{\prime}\left(A_{1}, A_{4}\right), & \Phi\left(\left\{v_{1}, v_{2}, v_{3}\right\}\right)=C^{\prime}\left(A_{0}, A_{4}\right),
\end{aligned}
$$

and the maps $\phi\left(s_{1}, s_{2}\right)$ for $s_{1} \leqq s_{2}$ in $K$ other than $\left\{v_{1}, v_{3}\right\}$ to be the maps induced 
by composing on either or both sides by appropriate $a_{i}$ 's (for example, if $s_{1}=\left\{v_{2}\right\}, s_{2}=\left\{v_{1}, v_{2}, v_{3}\right\}$, then $\phi\left(s_{1}, s_{3}\right): C^{\prime}\left(A_{1}, A_{3}\right) \rightarrow C^{\prime}\left(A_{0}, A_{4}\right)$ is the map $\phi\left(s_{1}, s_{3}\right)(g)=a_{4} g a_{1}$ for $\left.g \in C^{\prime}\left(A_{1}, A_{3}\right)\right)$, while $\phi\left(\left\{v_{1}, v_{3}\right\},\left\{v_{1}, v_{2}, v_{3}\right\}\right)\left(g_{1} g_{2}\right)=g_{2} g_{1}$ for $g_{1} \in C^{\prime}\left(A_{0}, A_{2}\right), g_{2} \in C^{\prime}\left(A_{2}, A_{4}\right)$ and

$$
\begin{aligned}
& \phi\left(v_{1},\left\{v_{1}, v_{3}\right\}\right)(g)=\left(g, a_{4} a_{3}\right) \text { for } g \in C^{\prime}\left(A_{0}, A_{2}\right), \\
& \phi\left(v_{3},\left\{v_{1}, v_{3}\right\}\right)(g)=\left(a_{2} a_{1}, g\right) \text { for } g \in C^{\prime}\left(A_{2}, A_{4}\right) .
\end{aligned}
$$

There is a map $f$ carried by $(\Phi, \phi)$ defined by the condition that for $s \in K$ the map $f(s)$ is the constant map of $|s|$ into a suitable product of composites of $a_{i}$ 's into $\Phi(s)$ (for example $f\left(v_{2}\right)$ is the constant map of $v_{2}$ to $a_{3} a_{2}, f\left(\left\{v_{1}, v_{2}\right\}\right)$ sends $\left|\left(v_{1}, v_{2}\right)\right|$ to the point $a_{3} a_{2} a_{1} \in C^{\prime}\left(A_{0}, A_{3}\right)$ and $f\left(\left\{v_{1}, v_{3}\right\}\right)$ sends $\left|\left(v_{1}, v_{3}\right)\right|$ to the point $\left(a_{2} a_{1}, a_{4} a_{3}\right) \in C^{\prime}\left(A_{0}, A_{2}\right) \times C^{\prime}\left(A_{2}, A_{4}\right)$.

$f$ is null-homotopic on $K^{0}$ if and only if the composites $a_{2} a_{1}, a_{3} a_{2}, a_{4} a_{3}$ are all null-homotopic. If this is so, then it can easily be seen that any null-homotopy of $f$ on $K^{0}$ can be extended over the 1-simplex $\left\{v_{1}, v_{3}\right\}$ so the obstruction to a null-homotopy of $f$ on $K^{1}$ is determined by its values on the oriented 1-cells $\left(v_{1}, v_{2}\right)$ and $\left(v_{2}, v_{3}\right)$. It follows from (5.1) that on $\left(v_{1}, v_{2}\right)$ this obstruction is $\left\langle a_{1}, a_{2}, a_{3}\right\rangle$ and on $\left(v_{2}, v_{3}\right)$ this obstruction is $\left\langle a_{2}, a_{3}, a_{4}\right\rangle$. However, as pointed out in Example (4.8) the indeterminacy of $O^{1}(f)$ is smaller than the sum of the indeterminacies of $\left\langle a_{1}, a_{2}, a_{3}\right\rangle$ and $\left\langle a_{2}, a_{3}, a_{4}\right\rangle$ so that the vanishing of both $\left\langle a_{1}, a_{2}, a_{3}\right\rangle$ and $\left\langle a_{2}, a_{3}, a_{4}\right\rangle$ is a necessary, but in general not a sufficient, condition for the existence of a null-homotopy of $f$ on $K^{1}$.

We now assume that $f$ is null-homotopic on $K^{1}$. Any null-homotopy of $f$ on $K^{1}$ has an obstruction to extending it over $K$ which is completely determined by its value on the oriented 2 -simplex $\left(v_{1}, v_{2}, v_{3}\right)$. This value is an element of $\pi_{2}\left(C^{\prime}\left(A_{0}, A_{4}\right)\right) \approx\left[S^{2} A_{0}, A_{4}\right]$. The tertiary operation $\left\langle a_{1}, a_{2}, a_{3}, a_{4}\right\rangle$ is defined to be the subset of $\left[S^{2} A_{0}, A_{4}\right]$ obtained by evaluating obstruction cocycles to extending null-homotopies of $f$ on $K^{1}$ on the oriented 2-simplex $\left(v_{1}, v_{2}, v_{3}\right)$.

We want to generalize the above to define $\left\langle a_{1}, a_{2}, \cdots, a_{n}\right\rangle$ for a sequence

$$
A_{0} \stackrel{a_{1}}{\longrightarrow} A_{1} \stackrel{a_{2}}{\longrightarrow} \cdots \stackrel{a_{n}}{\longrightarrow} A_{n}
$$

of continuous maps between based spaces. It is convenient to define $\left\langle a_{1}, a_{2}\right\rangle$ to be the homotopy class of the composite $a_{2} a_{1}$, and this is a primary operation. For $n>2$ let $K$ be the complex composed of an $(n-2)$-simplex with vertices $v_{1}, v_{2}, \cdots, v_{n-1}$ and all of its faces. For $1 \leqq i \leqq j \leqq n-1$ let $t(i, j)$ denote the simplex $\left\{v_{i}, v_{i+1}, \cdots, v_{j}\right\}$ of $K$. Then any simplex $s$ of $K$ has a unique representation in the form of a join

$$
s=t\left(i_{1}, j_{1}\right) t\left(i_{2}, j_{2}\right) \cdots t\left(i_{q}, j_{q}\right)
$$

where $j_{1}<i_{2}-1, j_{2}<i_{3}-1, \cdots, j_{q-1}<i_{q}-1$. We define a carrier $(\Phi, \phi)$ on $K$ by

$$
\Phi(s)=C^{\prime}\left(A_{i_{1}-1}, A_{j_{1}+1}\right) \times C^{\prime}\left(A_{i_{2}-1}, A_{j_{2}+1}\right) \times \cdots \times C^{\prime}\left(A_{i-1}, A_{j+1}\right)
$$


for $s$ represented as above. To define the maps $\phi\left(s, s^{\prime}\right)$ for $s \leqq s^{\prime}$ where $s^{\prime}$ has the representation

we let

$$
s^{\prime}=t\left(i_{1}^{\prime}, i_{1}^{\prime}\right) t\left(i_{2}^{\prime}, j_{2}^{\prime}\right) \cdots t\left(i_{r}^{\prime}, j_{r}^{\prime}\right)
$$

$$
\phi\left(s, s^{\prime}\right)\left(g_{1}, g_{2}, \cdots, g_{q}\right)=\left(g_{1}^{\prime}, g_{2}^{\prime}, \cdots, g_{r}^{\prime}\right)
$$

where each $g_{j}^{\prime}$ is an appropriate composite of $g_{i}$ 's and $a_{k}$ 's. For example,

$$
\begin{aligned}
\phi\left(v_{1},\left\{v_{1}, v_{2}\right\}\right)\left(g_{1}\right) & =\left(a_{3} g_{1}\right), \\
\phi\left(\left\{v_{1}, v_{4}\right\},\left\{v_{1}, v_{2}, v_{3}, v_{4}\right\}\right)\left(g_{1}, g_{2}\right) & =\left(g_{2} a_{3} g_{1}\right), \\
\phi\left(\left\{v_{1}, v_{3}, v_{4}\right\},\left\{v_{1}, v_{2}, v_{3}, v_{4}, v_{7}\right\}\right)\left(g_{1}, g_{2}\right) & =\left(g_{2} g_{1}, a_{8} a_{7}\right) .
\end{aligned}
$$

These maps $\phi\left(s, s^{\prime}\right)$ with $\Phi$ do define a carrier on $K$ and there is a map $f$ carried by $(\Phi, \phi)$ which assigns to $s=t\left(i_{1}, j_{1}\right) t\left(i_{2}, j_{2}\right) \cdots t\left(i_{q}, j_{q}\right)$ the constant map of $|s|$ to the point

$$
\left(a_{j_{1}+1} a_{j_{1}} \cdots a_{i_{1}}, a_{j_{2}+1} a_{j_{2}} \cdots a_{i_{2}}, \cdots, a_{j_{q}+1} a_{j_{q}} \cdots a_{i_{n}}\right) \in \Phi(s) .
$$

$f$ is null-homotopic on $K^{0}$ if and only if each composite $a_{j+1} a_{j}$ is null-homotopic (i.e., $\left\langle a_{j}, a_{j+1}\right\rangle=0$ ) for $1 \leqq j<n$. If this is so and $F$ is a null-homotopy of $f$ on $K^{0}$, then $F$ can be extended over any 1 -simplex $\left\{v_{i}, v_{j}\right\}$ with $i<j-1$ so the only 1-simplexes over which $F$ might not be extendable are of the form $\left\{v_{j}, v_{j+1}\right\}$ for some $j$ with $1 \leqq j<n-1$. There is a null-homotopy of $f$ on $\left\{v_{j}, v_{j+1}\right\}$ if and only if $\left\langle a_{j}, a_{j+1}, a_{j+2}\right\rangle$ vanishes. However, the vanishing of all $\left\langle a_{j}, a_{j+1}\right\rangle$ and $\left\langle a_{j}, a_{j+1}, a_{j+2}\right\rangle$ is not sufficient to insure that $f$ is null-homotopic on $K^{1}$. If $O^{1}(f)=0$, then $O^{2}(f)$ is defined and representative cocycles can be nonzero only on simplexes of the form $\left\{v_{j}, v_{j+1}, v_{j+2}\right\}$ for some $1 \leqq j<n-2$. If $f$ is null-homotopic on $\left\{v_{j}, v_{j+1}, v_{j+2}\right\}$, then $\left\langle a_{j}, a_{j+1}, a_{j+2}, a_{j+3}\right\rangle$ vanishes.

If $f$ is null-homotopic on $K^{n-3}$, then any null-homotopy of $f$ on $K^{n-3}$ has an obstruction to extension over $K$ which is completely determined by its value on the oriented $(n-2)$-simplex $\left(v_{1}, v_{2}, \cdots, v_{n-1}\right)$. This value is an element of

$$
\pi_{n-2}\left(\Phi\left(\left\{v_{1}, v_{2}, \cdots, v_{n-1}\right\}\right)\right) \approx\left[S^{n-2} A_{0}, A_{n}\right] \text {. }
$$

In this way $O^{n-2}(f)$ corresponds to a subset of $\left[S^{n-2} A_{0}, A_{n}\right]$, and we define the $(n-1)$-order operation $\left\langle a_{1}, a_{2}, \cdots, a_{n}\right\rangle$ to be this subset of $\left[S^{n-2} A_{0}, A_{n}\right]$. From this it follows that if $\left\langle a_{1}, a_{2}, \cdots, a_{n}\right\rangle$ is defined (i.e., if $f$ is null-homotopic on $K^{n-3}$ ), then all lower order operations $\left\langle a_{j}, a_{j+1}, \cdots, a_{j+q}\right\rangle$ for $q \leqq n-2$ are defined and vanish. However, if all these lower order operations are defined and vanish it does not follow that $\left\langle a_{1}, a_{2}, \cdots, a_{n}\right\rangle$ is defined.

The general properties of $\$ 5$ carry over to give obvious naturality and multilinearity properties of $\left\langle a_{1}, a_{2}, \cdots, a_{n}\right\rangle$ which generalize the properties of $\S 4$ of [14] for the secondary operation $\left\langle a_{1}, a_{2}, a_{3}\right\rangle$. 
Our next objective is to show howthe operation $\left\langle a_{1}, a_{2}, \cdots, a_{n}\right\rangle$ is related to lower order operations. Let

$$
A_{0} \stackrel{a_{1}}{\longrightarrow} A_{1} \stackrel{a_{2}}{\longrightarrow} \cdots \stackrel{a_{n}}{\longrightarrow} A_{n}
$$

be a sequence of continuous mappings between based spaces and let $h: A_{n-2} \rightarrow A_{n}$ be a null-homotopy of the composite $a_{n} a_{n-1}$. It was pointed out in $\S 1$ that such null-homotopies $h$ are in 1-1 correspondence with the set of maps $g: A_{n-2} \rightarrow E\left(a_{n}\right)$ such that $p g=a_{n-1}$. Let $K$ be the complex composed of all nonempty subsets of $\left\{v_{1}, v_{2}, \cdots, v_{n-1}\right\}$ and let $(\Phi, \phi)$ be the carrier on $K$ and $f$ the map carried by it used to define $\left\langle a_{1}, a_{2}, \cdots, a_{n}\right\rangle$. A null-homotopy $h$ of $a_{n} a_{n-1}$ can be regarded as a null-homotopy of $f$ on $v_{n-1}$.

THEOREM (6.1). With the notation above there is a null-homotopy of $f$ whose restriction to $v_{n-1}$ is $h$ if and only if for the sequence

$$
A_{0} \stackrel{a_{1}}{\longrightarrow} A_{1} \stackrel{a_{2}}{\longrightarrow} \cdots \stackrel{a_{n-2}}{\longrightarrow} A_{n-2} \stackrel{g}{\longrightarrow} E\left(a_{n}\right)
$$

$\left\langle a_{1}, a_{2}, \cdots, a_{n-2}, g\right\rangle$ is defined and vanishes.

Proof. Let $K^{\prime}$ be the complex composed of all nonempty subsets of $\left\{v_{1}, \cdots, v_{n-2}\right\}$ and let $\left(\Phi^{\prime}, \phi^{\prime}\right)$ be the carrier on $K^{\prime}$ and $f^{\prime}$ the map carried by it used to define $\left\langle a_{1}, a_{2}, \cdots, a_{n-2}, g\right\rangle$. If $s^{\prime}$ is any simplex of $K^{\prime}$ not containing $v_{n-2}$, then $\Phi^{\prime}\left(s^{\prime}\right)=\Phi\left(s^{\prime}\right)$ and $f^{\prime}\left(s^{\prime}\right)=f\left(s^{\prime}\right)$. If $s^{\prime}$ contains $v_{n-2}$, it follows from remarks made in $\S 1$ that a point of $\Phi^{\prime}\left(s^{\prime}\right)$ can be identified in a canonical way with a point of $\Phi\left(s^{\prime}\right)$ and a null-homotopy of the image of this point in $\Phi\left(s^{\prime} v_{n-1}\right)$ under the map $\phi\left(s^{\prime}, s^{\prime} v_{n-1}\right)$. Furthermore, under this correspondence $f^{\prime}\left(s^{\prime}\right)$ corresponds to $f\left(s^{\prime}\right)$ and the null-homotopy $h$. The theorem follows on observing that a null-homotopy of $f^{\prime}$ in $\left(\Phi^{\prime}, \phi^{\prime}\right)$ corresponds in exactly this way to a nullhomotopy of $f$ in $(\Phi, \phi)$ which is an extension of $h$ and conversely.

COROLlary (6.2). Given a sequence

$$
A_{0} \stackrel{a_{1}}{\longrightarrow} A_{1} \stackrel{a_{2}}{\longrightarrow} \cdots \stackrel{a_{n}}{\longrightarrow} A_{n}
$$

$\left\langle a_{1}, a_{2}, \cdots, a_{n}\right\rangle$ is defined and vanishes if and only if there is a commutative diagram

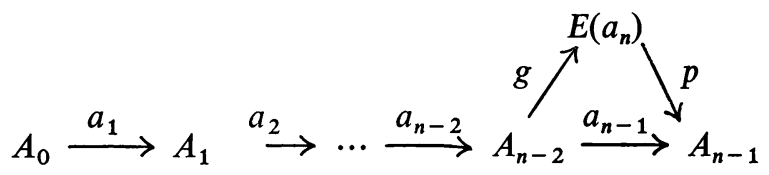

such that $\left\langle a_{1}, a_{2}, \cdots, a_{n-2}, g\right\rangle$ is defined and vanishes.

Proof. If $\left\langle a_{1}, a_{2}, \cdots, a_{n}\right\rangle$ is defined and vanishes, let $F$ be a null-homotopy of $f$ carried by $(\Phi, \phi)$ and let $g: A_{n-2} \rightarrow E\left(a_{n}\right)$ be the map corresponding to the 
null-homotopy $F\left(v_{n-1}\right)$ of $a_{n} a_{n-1}$. By (6.1) it follows that $\left\langle a_{1}, a_{2}, \cdots, a_{n-2}, g\right\rangle$ is defined and vanishes.

Conversely, if there is a $g$ such that the diagram above is commutative and $\left\langle a_{1}, a_{2}, \cdots, a_{n-2}, g\right\rangle$ is defined and vanishes, let $h$ be the null-homotopy of $a_{n} a_{n-1}$ corresponding to $g$. It follows from (6.1) that there is a null-homotopy $F$ of $f$ in $(\Phi, \phi)$ which extends $h$. Therefore, $\left\langle a_{1}, a_{2}, \cdots, a_{n}\right\rangle$ is defined and vanishes.

Given a sequence of continuous maps between based spaces (where $n \geqq 3$ )

$$
A_{0} \stackrel{a_{1}}{\longrightarrow} A_{1} \stackrel{a_{2}}{\longrightarrow} \cdots \stackrel{a_{n}}{\longrightarrow} A_{n}
$$

a splitting of this sequence is a commutative diagram of continuous maps between based spaces

$$
\begin{aligned}
& b_{2} \nearrow^{B_{2}} \backslash c_{2} b_{3} \int^{B_{3}} \downarrow_{3} \downarrow^{B_{n-1}} c_{n-1} \\
& A_{0} \stackrel{a_{1}}{\longrightarrow} A_{1} \stackrel{a_{2}}{\longrightarrow} A_{2} \stackrel{a_{3}}{\longrightarrow} \cdots \stackrel{a_{n-1}}{\longrightarrow} A_{n-1} \stackrel{a_{n}}{\longrightarrow} A_{n}
\end{aligned}
$$

such that the composites $b_{2} a_{1}, b_{3} c_{2}, \cdots, b_{i+1} c_{i}, \cdots, b_{n-1} c_{n-2}, a_{n} c_{n-1}$ are all nullhomotopic. If such a splitting exists we say that the original sequence

$$
A_{0} \stackrel{a_{1}}{\longrightarrow} A_{1} \stackrel{a_{2}}{\longrightarrow} \cdots \stackrel{a_{n}}{\longrightarrow} A_{n}
$$

splits. If $n=2$, we also say $A_{0} \stackrel{a_{1}}{\rightarrow} A_{1} \stackrel{a_{2}}{\rightarrow} A_{2}$ splits if the composite $a_{2} a_{1}$ is null-homotopic.

THEOREM (6.3). A sequence

$$
A_{0} \stackrel{a_{1}}{\longrightarrow} A_{1} \stackrel{a_{2}}{\longrightarrow} \cdots \stackrel{a_{n}}{\longrightarrow} A_{n}
$$

splits if and only if $\left\langle a_{1}, a_{2}, \cdots, a_{n}\right\rangle$ is defined and vanishes.

Proof. We prove the theorem by induction on $n$. By definition the theorem is true for $n=2$. Let $n>2$ and assume the following diagram is a splitting.

$$
\begin{aligned}
A_{0} \stackrel{a_{1}}{\longrightarrow} A_{1} \stackrel{a_{2}}{\longrightarrow} A_{2} \longrightarrow \cdots \longrightarrow A_{n-1} \stackrel{a_{2}}{\longrightarrow} A_{n} . & \bigcup_{n-1}^{B_{n-1}} c_{n-1}
\end{aligned}
$$

Then the following diagram 


$$
\begin{aligned}
b_{2} \uparrow \downarrow^{B_{2}} c_{2} & \rfloor^{B_{n-2}} \\
A_{0} \stackrel{a_{1}}{\longrightarrow} A_{1} \stackrel{a_{2}}{\longrightarrow} A_{2} \longrightarrow & \longrightarrow \stackrel{a_{n-2}}{\longrightarrow} A_{n-2} \stackrel{b_{n-1}}{\longrightarrow} B_{n-1}
\end{aligned}
$$

is a splitting of the sequence

$$
A_{0} \stackrel{a_{1}}{\longrightarrow} A_{1} \stackrel{a_{2}}{\longrightarrow} \cdots \stackrel{a_{n-2}}{\longrightarrow} A_{n-2} \stackrel{b_{n-1}}{\longrightarrow} B_{n-1} .
$$

Therefore, by the inductive assumption $\left\langle a_{1}, \cdots, a_{n-2}, b_{n-1}\right\rangle$ is defined and vanishes. Since $a_{n} c_{n-1}: B_{n-1} \rightarrow A_{n}$ is null-homotopic, there exists a continuous map $g^{\prime}: B_{n-1} \rightarrow E\left(a_{n}\right)$ such that commutativity holds in the diagram

$$
\begin{aligned}
b_{n-1}{ }^{B_{n-1}} \stackrel{g^{\prime}}{\longrightarrow} E\left(a_{n}\right) \\
\downarrow^{c_{n-1}} \downarrow p \\
A_{n-2} \stackrel{a_{n-1}}{\longrightarrow} A_{n-1} .
\end{aligned}
$$

Hence, if we define $g: A_{n-2} \rightarrow E\left(a_{n}\right)$ to be the composite $g^{\prime} b_{n-1}$, we have the commutative diagram

$$
A_{0} \stackrel{a_{1}}{\longrightarrow} A_{1} \stackrel{a_{2}}{\longrightarrow} \ldots \stackrel{a_{n-2}}{\longrightarrow} A_{n-2} \stackrel{a_{n-1}}{\longrightarrow} A_{n-1} .
$$

By the naturality of the operation

$$
\left\langle a_{1}, \cdots, a_{n-2}, g\right\rangle=\left\langle a_{1}, \cdots, a_{n-2}, g^{\prime} b_{n-1}\right\rangle=g_{\#}^{\prime}\left\langle a_{1}, \cdots, a_{n-2}, b_{n-1}\right\rangle
$$

so $\left\langle a_{1}, \cdots, a_{n-2}, g\right\rangle$ is defined and vanishes. By (6.2) it follows that $\left\langle a_{1}, a_{2}, \cdots, a_{n}\right\rangle$ is defined and vanishes which proves half of the theorem.

For the other half assume $\left\langle a_{1}, a_{2}, \cdots, a_{n}\right\rangle$ is defined and vanishes. By (6.2) there exists a commutative diagram

$$
A_{0} \stackrel{1}{\longrightarrow} A_{1} \stackrel{a_{2}}{\longrightarrow} \cdots \stackrel{a_{n-2}}{\longrightarrow} A_{n-2} \stackrel{a_{n-1}}{\longrightarrow} A_{n-1}
$$

such that $\left\langle a_{1}, a_{2}, \cdots, a_{n-2}, g\right\rangle$ is defined and vanishes. By the inductive assumption there is a splitting 


$$
\begin{gathered}
b_{2} \bigwedge^{B_{2}} \downarrow c_{2} \\
A_{0} \stackrel{a_{1}}{\longrightarrow} A_{1} \stackrel{a_{2}}{\longrightarrow} A_{2} \longrightarrow \ldots \stackrel{B_{n-2}}{\longrightarrow} \downarrow_{n-2}^{\longrightarrow} A_{n-2} \stackrel{g}{\longrightarrow} E\left(a_{n}\right)
\end{gathered}
$$

of the sequence

$$
A_{0} \stackrel{a_{1}}{\longrightarrow} A_{2} \stackrel{a_{2}}{\longrightarrow} \ldots \stackrel{a_{n-2}}{\longrightarrow} A_{n-2} \stackrel{g}{\longrightarrow} E\left(a_{n}\right) .
$$

Then the following diagram

$$
\begin{aligned}
& \left.b_{2} \uparrow^{B_{2}} \downarrow c_{2} \quad \downarrow^{B_{n-2}} g\right\rceil^{E\left(a_{n}\right)} p \\
& A_{0} \stackrel{a_{1}}{\longrightarrow} A_{1} \stackrel{a_{2}}{\longrightarrow} A_{2} \longrightarrow \ldots \stackrel{a_{n-2}}{\longrightarrow} A_{n-2} \stackrel{a_{n-1}}{\longrightarrow} A_{n-1} \stackrel{a_{n}}{\longrightarrow} A_{n}
\end{aligned}
$$

is a splitting of the original sequence, and this completes the proof.

Assume we have a sequence

$$
A_{0} \stackrel{a_{1}}{\longrightarrow} A_{1} \stackrel{a_{2}}{\longrightarrow} \ldots \stackrel{a_{n}}{\longrightarrow} A_{n}
$$

such that $\left\langle a_{1}, a_{2}, \ldots, a_{n}\right\rangle$ is defined and vanishes. Let $K$ be the complex composed of all nonempty subsets of $\left\{v_{1}, v_{2}, \ldots, v_{n-1}\right\}$ and let $(\Phi, \phi)$ be the carrier on $K$ and $f$ the map carried by it used to define $\left\langle a_{1}, a_{2}, \ldots, a_{n}\right\rangle$. By the definition of $\left\langle a_{1}, a_{2}, \ldots, a_{n}\right\rangle$ the vanishing of $\left\langle a_{1}, a_{2}, \ldots, a_{n}\right\rangle$ is equivalent to the existence of a null-homotopy of $f$. Our next result gives a description of such null-homotopies.

LemMa (6.4). A null-homotopy of $f$ carried by $(\Phi, \phi)$ corresponds to a sequence of maps $g_{i}(1 \leqq i \leqq n-1)$ such that the following diagram is commutative

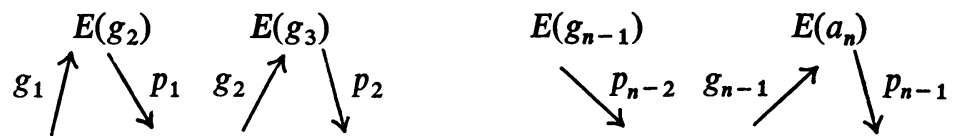

$$
\begin{aligned}
& A_{0} \stackrel{a_{1}}{\longrightarrow} A_{1} \stackrel{a_{2}}{\longrightarrow} A_{2} \longrightarrow \ldots \stackrel{a_{n-2}}{\longrightarrow} A_{n-2} \stackrel{a_{n-1}}{\longrightarrow} A_{n-1} \stackrel{a_{n}}{\longrightarrow} A_{n} \text {. }
\end{aligned}
$$

Proof. It follows from the proof of (6.1) that a null-homotopy of $f$ carried by $(\Phi, \phi)$ corresponds to a map $g: A_{n-2} \rightarrow E\left(a_{n}\right)$ such that $p_{n-1} g=a_{n-1}$ and a null-homotopy of the map $f^{\prime}$ carried by $\left(\Phi^{\prime}, \phi^{\prime}\right)$ used to define $\left\langle a_{1}, a_{2}, \ldots, a_{n-2}, g\right\rangle$ for the sequence

$$
A_{0} \stackrel{a_{1}}{\longrightarrow} A_{1} \stackrel{a_{2}}{\longrightarrow} \ldots \stackrel{a_{n-2}}{\longrightarrow} A_{n-2} \stackrel{g}{\longrightarrow} E\left(a_{n}\right)
$$


setting $g_{n-1}=g$ we see from the above remark that the theorem follows by an easy induction on $n$.

With the same notation as above let $F$ be a null-homotopy of $f$ carried by $(\Phi, \phi)$ and let the following commutative diagram correspond to it

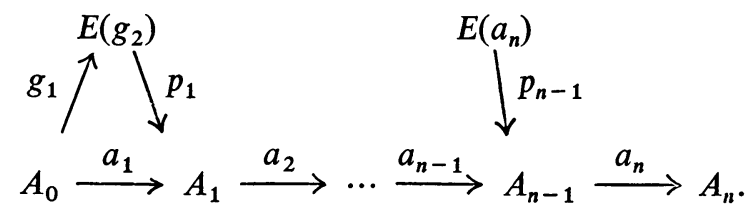

Let $X$ be an arbitrary based space and let $a_{0}: X \rightarrow A_{0}$ be a continuous map. Then we have the sequence

$$
X \stackrel{a_{0}}{\longrightarrow} A_{0} \stackrel{a_{1}}{\longrightarrow} \cdots \stackrel{a_{n}}{\longrightarrow} A_{n}
$$

so if $\bar{K}$ denotes the complex composed of all nonempty subsets of $\left\{v_{0}, v_{1}, \cdots, v_{n-1}\right\}$ there is a carrier $(\bar{\Phi}, \bar{\phi})$ on $R$ and a map $\bar{f}$ carried by it used to define $\left\langle a_{0}, a_{1}, \cdots, a_{n}\right\rangle$. The complex $K$ is a subcomplex of $K$ and on $K$ the carrier $(\bar{\Phi}, \bar{\phi})$ is just $(\Phi, \phi)$ and $\bar{f}$ is just $f$. Hence, $F$ is a null-homotopy of $\bar{f}$ on $K$, and we can define higher order operations by considering the successive obstructions to extending the null-homotopy $F$ to a null-homotopy of all of $\bar{f}$. If $F$ has been extended to a null-homotopy $F$ on $\bar{K}^{k-1}$, then the only $k$-dimensional simplex of $\bar{K}$ on which $c^{k}(F)$ can have a nonzero value is the simplex $\left\{v_{0}, v_{1}, \cdots, v_{k}\right\}$. Therefore, $c^{k}(F)$ is completely determined by its value on the oriented $k$-cell $\left(v_{0}, v_{1}, \cdots, v_{k}\right)$, and this value is an element of

$$
\pi_{k}\left(\bar{\Phi}\left(\left\{v_{0}, v_{1}, \cdots, v_{k}\right\}\right)\right) \approx\left[S^{k} X, A_{k+1}\right] \approx\left[X, \Omega^{k} A_{k+1}\right] .
$$

Hence, if the $(k+1)$ st order operation is defined it can be regarded as a subset of $\left[X, \Omega^{k} A_{k+1}\right]$, and if it vanishes, the $(k+2)$ nd order operation is defined. These subsets form subsets of $\left\langle a_{0}, a_{1}, \cdots, a_{k+1}\right\rangle$, the latter being the obstruction to a null-homotopy of $\bar{f}$ on the simplex $\left\{v_{0}, v_{1}, \cdots, v_{k}\right\}$, but have smaller indeterminacy because we are restricting attention only to null-homotopies of $f$ which are extensions of $F$.

These operations can be defined directly by means of the commutative diagrams corresponding to $F$. Thus we have the commutative diagram

$$
X \stackrel{a_{0}}{\longrightarrow} A_{0} \stackrel{a_{1}}{\longrightarrow} A_{1} .
$$

The first operation is represented by the composite $a_{1} a_{0}$. If this is null-homotopic, since $p_{1}$ is a fiber map, the map $g_{1} a_{0}$ is homotopic to a map $h$ of $X$ into 
the fiber $\Omega E\left(g_{3}\right)$ of $p_{1}$. Now $\Omega p_{2}: \Omega E\left(g_{3}\right) \rightarrow \Omega A_{2}$ is again a fibration and the collection of all homotopy classes of composites $\left(\Omega p_{2}\right) h$ for all such maps $h: X \rightarrow \Omega E\left(g_{3}\right)$ is the second order operation. If it vanishes, there exist maps $X \rightarrow \Omega^{2} E\left(g_{4}\right)$, etc. In the stable range these higher order operations can be seen to correspond to the higher order operations considered by Peterson [10] and to the higher order differentials in the spectral sequence of Adams [2].

\section{REFERENCES}

1. J. F. Adams, On the non-existence of elements of Hopf invariant one, Ann. of Math. (2) 72 (1960), 20-104. 1961.

2. - Stable homotopy theory, Mimeographed, University of California at Berkeley,

3. C. R. Curjel, Über die Homotopie- und Cohomologiegruppen von Abbildungen, Comment. Math. Helv. 35 (1961), 233-262.

4. A. Dold, Zur Homotopietheorie der Kettenkomplexe, Math. Ann. 148 (1960), 278-298.

5. W. S. Massey, Some higher order cohomology operations, Symposium Internacional de Topologia Algebraica, pp. 145-154, La Universidad Nacional Autónama de México and UNESCO, Mexico City, 1958.

6. W. S. Massey and H. Uehara, The Jacobi identity for Whitehead products, Algebraic Geometry and Topology (a symposium in honor of S. Lefschetz), pp. 361-377, Princeton Univ. Press, Princeton, N. J., 1957.

7. P. Olum, Non-abelian cohomology and Van Kampen's theorem, Ann. of Math. (2) 68 (1958), 658-668.

8. - Obstructions to extensions and homotopies, Ann. of Math. (2) 52 (1950), 1-50.

9. F. P. Peterson, Functional cohomology operations, Trans. Amer. Math. Soc. 86 (1957), 197-211.

10. - Functional higher order cohomology operations, Symposium Internacional de Topologia Algebraica, pp. 159-164, Mexico City, 1958.

11. F. P. Peterson and N. Stein, Secondary cohomology operations: two formulas, Amer. J. Math. 81 (1959), 281-305.

12. F. P. Peterson and E. Thomas, A note on non-stable cohomology operations, Bol. Soc. Mat. Mexicana 3 (1958), 13-18.

13. D. Puppe, Homotopiemengen und ihre induzierten Abbildungen. I, Math. Z. 69 (1958), 299-344.

14. E. Spanier, Secondary operations on mappings and cohomology, Ann. of Math. (2) 75 (1962), 260-282.

15. ——, Quasi-topologies, Duke Math. J. 30 (1963), 1-14.

16. E. Spanier and J. H. C. Whitehead, Carriers and S-theory, Algebraic Geometry and Topology (a symposium in honor of S. Lefschetz), pp. 330-360, Princeton Univ. Press, Princeton, N. J., 1957.

17. N. E. Steenrod, Cohomology invariants of mappings, Ann. of Math. (2) 50 (1949), 954-988.

18. H. Toda, Generalized Whitehead products and homotopy groups of spheres, J. Inst. Polytech. Osaka City Univ. Ser. A 3 (1952), 43-82.

19. - Composition methods in homotopy groups of spheres, Annals of Mathematics Studies No. 49, Princeton Univ. Press, Princeton, N. J., 1962. 